\title{
A GENERALIZATION OF COLMEZ-GREENBERG-STEVENS FORMULA
}

\author{
BINGYONG XIE
}

\author{
Department of Mathematics, East China Normal University, Shanghai, China. \\ E-mail: byxie@math.ecnu.edu.cn \\ \|\|\|\|
}

\begin{abstract}
In this paper we study the derivatives of Frobenius and the derivatives of Hodge-Tate weights for families of Galois representations with triangulations. We give a generalization of the Fontaine-Mazur $\mathcal{L}$-invariant and use it to build a formula which is a generalization of the Colmez-Greenberg-Stevens formula.
\end{abstract}

\section{Introduction}

In their remarkable paper [10], Mazur, Tate and Teitelbaum proposed a conjectural formula for the derivative at $s=1$ of the $p$-adic $L$-function of an elliptic curve $E$ over $\mathbf{Q}$ when $p$ is a prime of split multiplicative reduction. An important quantity in this formula is the so called $\mathcal{L}$-invariant, namely $\mathcal{L}(E)=\log _{p}\left(q_{E}\right) / v_{p}\left(q_{E}\right)$ where $q_{E} \in \mathbf{Q}_{p}^{\times}$is the Tate period for $E$. This conjectural formula was proved by Greenberg and Stevens [8] using Hida's families. Indeed, for the weight 2 newform $f$ attached to $E$, there exists a family of $p$-adic ordinary Hecke eigenforms containing $f$. A key formula they proved is

$$
\mathcal{L}(E)=-2 \frac{\alpha^{\prime}(f)}{\alpha(f)}
$$

where $\alpha$ is the function of $U_{p^{-}}$-eigenvalues of the eigenforms in the Hida family. On the other hand, they showed that $-2 \frac{\alpha^{\prime}(f)}{\alpha(f)}$ is equal to $\frac{L_{p}^{\prime}(f, 1)}{L(f, 1)}$. Combining

Received November 23, 2020 and in revised form December 16, 2020.

AMS Subject Classification: 11F80, $11 \mathrm{~F} 85$.

Key words and phrases: Frobenius, Hodge-Tate weight, $\mathcal{L}$-invariant.

This paper is supported by Science and Technology Commission of Shanghai Municipality (grant no. 13dz2260400) and the National Natural Science Foundation of China (grant no. 11671137). 
these two facts they obtained the conjectural formula.

In this paper we will focus on (1.1) which was later generalized by Colmez [6] to the non-ordinary setting. We state Colmez's result below.

Theorem 1.1 ([6]). Suppose that, at each closed point $z$ of $\operatorname{Max}(S)$ one of the Hodge-Tate weight of $\mathcal{V}_{z}$ is 0 , and there exists $\alpha \in S$ such that $\left(\mathbf{B}_{\text {cris }, S}^{\varphi=\alpha} \widehat{\otimes}_{S} \mathcal{V}\right)^{G_{\mathbf{Q}_{p}}}$ is locally free of rank 1 over $S$. Suppose $z_{0}$ is a closed point of $\operatorname{Max}(S)$ such that $\mathcal{V}_{z_{0}}$ is semistable with Hodge-Tate weights $\| 0$ and $k \geq 1$. Then the differential

$$
\frac{\mathrm{d} \alpha}{\alpha}-\frac{1}{2} \mathcal{L} \mathrm{d} \kappa+\frac{1}{2} \mathrm{~d} \delta
$$

is zero at $z_{0}$, where $\mathcal{L}$ is the Fontaine-Mazur $\mathcal{L}$-invariant of $\mathcal{V}_{z_{0}}$.

See [6] for the precise meanings of $\kappa$ and $\delta$. Roughly speaking, $\mathrm{d} \delta$ is the derivative of Frobenius, and $\mathrm{d} \kappa$ is the derivative of Hodge-Tate weights.

The condition that " $\left(\mathbf{B}_{\text {cris }, S}^{\varphi=\alpha} \widehat{\otimes}_{S} \mathcal{V}\right)^{G_{\mathbf{Q}_{p}}}$ is locally free of rank 1 over $S$ " in Theorem 1.1 is equivalent to that $\mathcal{V}$ admits a triangulation [5]. So, Theorem 1.1 means that the derivatives of Frobenius and the derivatives of Hodge-Tate weights of a family of 2-dimensional representations of $G_{\mathbf{Q}_{p}}$ with a triangulation satisfy a non-trivial relation at each semistable (but non-crystalline) point.

Colmez's theorem was generalized by Zhang [14] for families of 2- dimensional Galois representations of $G_{K}\left(K\right.$ a finite extension of $\left.\mathbf{Q}_{p}\right)$ and Pottharst [12] who considered families of (not necessarily étale) $(\varphi, \Gamma)$-modules of rank 2 instead of families of 2-dimensional Galois representations.

In this paper we give a generalization of Colmez's theorem which includes the above generalizations as special cases.

Fix a finite extension $K$ of $\mathbf{Q}_{p}$. What we work with is a family of $K$ $B$-pair (called $S$ - $B$-pair in our context) that is locally triangulable. We will provide conditions for Fontaine-Mazur $\mathcal{L}$-invariant to be defined. Note that, the $\mathcal{L}$-invariant is now a vector with component number equal to $\left[K: \mathbf{Q}_{p}\right]$.

\footnotetext{
${ }^{1}$ In this paper, the Hodge-Tate weights are defined to be minus the generalized eigenvalues of Sen's operators. In particular the Hodge-Tate weight of the cyclotomic character $\chi_{\text {cyc }}$ is -1 .
} 
Theorem 1.2. Let $W$ be an $S$-B-pair that is semistable at a point $z \in$ $\operatorname{Max}(S)$. Suppose that $W$ is locally triangulable at $z$ with the local triangulation parameters $\left(\delta_{1}, \ldots, \delta_{n}\right)$. Assume that for $D_{z}$, the filtered $E-(\varphi, N)$ module attached to $W_{z}$, the Fontaine-Mazur $\mathcal{L}$-invariant $\overrightarrow{\mathcal{L}}_{s, t}($ see Definition 6.5) can be defined for $s, t \in\{1,2, \ldots, n\}$. Then

$$
\frac{1}{\left[K: \mathbf{Q}_{p}\right]}\left(\frac{\mathrm{d} \delta_{t}(p)}{\delta_{t}(p)}-\frac{\mathrm{d} \delta_{s}(p)}{\delta_{s}(p)}\right)+\overrightarrow{\mathcal{L}}_{s, t} \cdot\left(\mathrm{d} \vec{w}\left(\delta_{t}\right)-\mathrm{d} \vec{w}\left(\delta_{s}\right)\right)=0 .
$$

Here, $\vec{w}\left(\delta_{i}\right)$ is the Hodge-Tate weight of the character $\delta_{i}$.

In [13] we proved Theorem 1.2 for a special case, where we consider the case of $K=\mathbf{Q}_{p}$ and demand that the Frobenius is simisimple at $z$. The motivation and some potential applications of our theorem was also discussed in [13].

Our paper is orginized as follows. In Section 2 we recall the theory of $B$-pairs built by Berger. Then in Section 3 we extend a part of this theory to families of $B$-pairs, and discuss the relation between triangulations of semistable $B$-pairs and refinements of their associated filtered $(\varphi, N)$ modules. In Section 4 we compare cohomology groups of $(\varphi, \Gamma)$-modules and those of $B$-pairs, and then attach a 1-cocycle to each infinitesimal deformation of a $B$-pair. In Section 5 we use the reciprocity law to build an auxiliary formula for $L$-invariants. The $L$-invariant is defined in Section 6 . In Section 7 we prove a formula called "projection vanishing property" for the above 1-cocycle. Finally in Section 8 we use the auxiliary formula in Section 5 and the projection vanishing property to deduce Theorem 1.2 .

\section{Notations}

Let $K$ be a finite extension of $\mathbf{Q}_{p}, G_{K}$ the absolute Galois group $\operatorname{Gal}(\bar{K} / K)$. Let $K_{0}$ be the maximal absolutely unramified subfield of $K$. Let $G_{K}^{\text {ab }}$ denote the maximal abelian quotient of $G_{K}$.

Let $\chi_{\mathrm{cyc}}$ be the cyclotomic character of $G_{K}, H_{K}$ the kernel of $\chi_{\mathrm{cyc}}$ and $\Gamma_{K}$ the quotient $G_{K} / H_{K}$. Then $\chi_{\text {cyc }}$ induces an isomorphism from $\Gamma_{K}$ onto an open subgroup of $\mathbf{Z}_{p}^{\times}$.

Let $E$ be a finite extension of $K$ such that all embeddings of $K$ into an algebraic closure of $E$ are contained in $E, \operatorname{Emb}(K, E)$ the set of embeddings 
of $K$ into $E$. We consider $E$ as a coefficient field and let $G_{K}$ acts trivially on $E$.

Let $\operatorname{rec}_{K}$ be the reciprocity map of local class field theory such that $\operatorname{rec}_{K}\left(\pi_{K}\right)$ is a lifting of the inverse of $q$ th power Frobenius of $k$, where $\pi_{K}$ is a uniformizing element of $K$ and $k$ is the residue field of $K$ with cardinal number $q$. Note that the image of $\operatorname{rec}_{K}$ coincides with the image of the Weil group $W_{K} \subset G_{K}$ by the quotient map $G_{K} \rightarrow G_{K}^{\text {ab }}$. Let $\operatorname{rec}_{K}^{-1}: W_{K} \rightarrow K^{\times}$ be the converse map of $\operatorname{rec}_{K}$.

\section{2. $\left(\varphi, \Gamma_{K}\right)$-modules and $B$-pairs}

\subsection{Fontaine's rings}

We recall the construction of Fontaine's period rings. Please consult [7, 2] for more details.

Let $\mathbf{C}_{p}$ be a completed algebraic closure of $\mathbf{Q}_{p}$ with valuation subring ${ }^{{ }^{\circ}} \mathbf{C}_{p}$ and $p$-adic valuation $v_{p}$ normalized such that $v_{p}(p)=1$.

Let $\widetilde{\mathbf{E}}$ be $\left\{\left(x^{(i)}\right)_{i \geq 0} \mid x^{(i)} \in \mathbf{C}_{p},\left(x^{(i+1)}\right)^{p}=x^{(i)} \forall i \in \mathbf{N}\right\}$, and let $\widetilde{\mathbf{E}}^{+}$be the subset of $\widetilde{\mathbf{E}}$ such that $x^{(0)} \in \mathfrak{o}_{\mathbf{C}_{p}}$. If $x, y \in \widetilde{\mathbf{E}}$, we define $x+y$ and $x y$ by

$$
(x+y)^{(i)}=\lim _{j \rightarrow \infty}\left(x^{(i+j)}+y^{(i+j)}\right)^{p^{j}}, \quad(x y)^{(i)}=x^{(i)} y^{(i)} .
$$

Then $\widetilde{\mathbf{E}}$ is a field of characteristic $p$. Define a function $v_{\mathbf{E}}: \widetilde{\mathbf{E}} \rightarrow \mathbf{R} \cup\{+\infty\}$ by putting $v_{\mathbf{E}}\left(\left(x^{(n)}\right)\right)=v_{p}\left(x^{(0)}\right)$. This is a valuation for which $\widetilde{\mathbf{E}}$ is complete and $\widetilde{\mathbf{E}}^{+}$is the ring of integers in $\widetilde{\mathbf{E}}$. If we let $\varepsilon=\left(\varepsilon^{(n)}\right)$ be an element of $\widetilde{\mathbf{E}}^{+}$with $\epsilon^{(0)}=1$ and $\epsilon^{(1)} \neq 1$, then $\widetilde{\mathbf{E}}$ is a completed algebraic closure of $\mathbf{F}_{p}((\varepsilon-1))$. Put $\omega=[\varepsilon]-1$. Let $\tilde{p}$ be an element of $\widetilde{\mathbf{E}}$ such that $\tilde{p}^{(0)}=p$.

Let $\widetilde{\mathbf{A}}^{+}$be the ring $\mathbf{W}\left(\widetilde{\mathbf{E}}^{+}\right)$of Witt vectors with coefficients in $\widetilde{\mathbf{E}}^{+}, \widetilde{\mathbf{A}}$ the ring of Witt vectors $\mathbf{W}(\widetilde{\mathbf{E}})$, and $\widetilde{\mathbf{B}}^{+}=\widetilde{\mathbf{A}}[1 / p]$. The map

$$
\theta: \widetilde{\mathbf{B}}^{+} \rightarrow \mathbf{C}_{p}, \quad \sum_{n \gg-\infty} p^{k}\left[x_{k}\right] \mapsto \sum_{n \gg-\infty} p^{k} x_{k}^{(0)}
$$

is surjective. Let $\mathbf{B}_{\mathrm{dR}}^{+}$be the $\operatorname{ker}(\theta)$-adic completion of $\widetilde{\mathbf{B}}^{+}$. Then $t_{\mathrm{cyc}}=$ $\log [\varepsilon]$ is an element of $\mathbf{B}_{\mathrm{dR}}^{+}$, and put $\mathbf{B}_{\mathrm{dR}}=\mathbf{B}_{\mathrm{dR}}^{+}\left[1 / t_{\mathrm{cyc}}\right]$. There is a filtration Fil ${ }^{\bullet}$ on $\mathbf{B}_{\mathrm{dR}}$ such that $\mathrm{Fil}^{i} \mathbf{B}_{\mathrm{dR}}=\bigoplus_{j \geq i} \mathbf{B}_{\mathrm{dR}}^{+} t_{\mathrm{cyc}}^{j}$. 
Let $\mathbf{B}_{\max }^{+}$be the subring of $\widetilde{\mathbf{B}}^{+}$consisting of elements of the form $\sum_{n \geq 0} b_{n}([\tilde{p}] / p)^{n}$, where $b_{n} \in \widetilde{\mathbf{B}}^{+}$and $b_{n} \rightarrow 0$ when $n \rightarrow+\infty$. Put $\mathbf{B}_{\max }=$ $\mathbf{B}_{\max }^{+}\left[1 / t_{\mathrm{cyc}}\right] ; \mathbf{B}_{\max }$ is equipped with a $\varphi$-action. Put $\mathbf{B}_{\log }=\mathbf{B}_{\max }[\log [\tilde{p}]] ;$ $\mathbf{B}_{\log }$ is equipped with a $\varphi$-action and a monodromy $N ; \mathbf{B}_{\log }^{N=0}=\mathbf{B}_{\max } ; \mathbf{B}_{\log }$ is a subring of $\mathbf{B}_{\mathrm{dR}}$. Put $\mathbf{B}_{e}=\mathbf{B}_{\max }^{\varphi=1}$. We have the following fundamental exact sequence

$$
0 \longrightarrow \mathbf{Q}_{p} \longrightarrow \mathbf{B}_{e} \longrightarrow \mathbf{B}_{\mathrm{dR}} / \mathbf{B}_{\mathrm{dR}}^{+} \longrightarrow 0
$$

If $r$ and $s$ are two elements in $\mathbf{N}[1 / p] \cup\{+\infty\}$, we put $\widetilde{\mathbf{A}}^{[r, s]}=\widetilde{\mathbf{A}}^{+}\left\{\frac{p}{\left[\bar{\omega}^{r}\right]}\right.$, $\left.\frac{\left[\bar{\omega}^{s}\right]}{p}\right\}$ and $\widetilde{\mathbf{B}}^{[r, s]}=\widetilde{\mathbf{A}}^{[r, s]}[1 / p]$ with the convention that $p /\left[\bar{\omega}^{+\infty}\right]=1 /[\bar{\omega}]$ and $\left[\bar{\omega}^{+\infty}\right] / p=0$. We equip these rings with the $p$-adic topology. There are natural continuous $G_{K}$-actions on $\widetilde{A}_{[r, s]}$ and $\widetilde{B}_{[r, s]}$. Frobenius induces isomorphisms $\varphi: \widetilde{A}_{[r, s]} \stackrel{\sim}{\rightarrow} \widetilde{A}_{[p r, p s]}$ and $\varphi: \widetilde{B}_{[r, s]} \stackrel{\sim}{\rightarrow} \widetilde{B}_{[p r, p s]}$. If $r \leq r_{0} \leq s_{0} \leq$ $s$, then we have the $G_{K^{-}}$-equivariant injective natural map $\widetilde{A}_{[r, s]} \hookrightarrow \widetilde{A}_{\left[r_{0}, s_{0}\right]}$. For $r>0$ we put $\widetilde{\mathbf{B}}_{\text {rig }}^{\dagger, r}=\bigcap_{s \in[r,+\infty)} \widetilde{B}_{[r, s]}$ (equipped with certain Frechet topology) and $\widetilde{\mathbf{B}}_{\text {rig }}^{\dagger}=\cup_{r>0} \widetilde{\mathbf{B}}_{\text {rig }}^{\dagger, r}$ (equipped with the inductive limit topology). Frobenius induces isomorphisms $\varphi: \widetilde{\mathbf{B}}_{\text {rig }}^{\dagger, r} \stackrel{\sim}{\rightarrow} \widetilde{\mathbf{B}}_{\text {rig }}^{\dagger, p r}$ and $\varphi: \widetilde{\mathbf{B}}_{\text {rig }}^{\dagger} \stackrel{\sim}{\rightarrow} \widetilde{\mathbf{B}}_{\text {rig }}^{\dagger}$.

Put

$$
\left.A_{K_{0}^{\prime}}=\left\{\sum_{k \geq-\infty}^{+\infty} a_{k} \omega^{k} \mid a_{k} \in \mathfrak{o}_{K_{0}^{\prime}}, a_{k} \rightarrow 0 \text { when } k \rightarrow-\infty\right)\right\}
$$

and $B_{K_{0}^{\prime}}=A_{K_{0}^{\prime}}[1 / p]$. Here $K_{0}^{\prime}$ is the maximal absolutely unramified subfield of $K_{\infty}=K\left(\mu_{p^{\infty}}\right)$. Then $A_{K_{0}^{\prime}}$ is a complete discrete valuation ring with $p$ as a prime element, and $B_{K_{0}^{\prime}}$ is the fractional field of $A_{K_{0}^{\prime}}$. The $G_{K^{-}}$-action and $\varphi$ preserve $A_{K_{0}^{\prime}}: \varphi(\omega)=(1+\omega)^{p}-1$ and $g(\omega)=(1+\omega)^{\chi_{\text {cyc }}(g)}-1$. Let $\mathbf{A}$ be the $p$-adic completion of the maximal unramified extension of $A_{K_{0}^{\prime}}$ in $\widetilde{\mathbf{A}}$, $\mathbf{B}$ its fractional field. Then $\varphi$ and the $G_{K^{-}}$action preserve $\mathbf{A}$ and $\mathbf{B}$.

We put $\mathbf{B}_{K}=\mathbf{B}^{H_{K}}$ and $\mathbf{B}_{K}^{\dagger, r}=\mathbf{B}_{K} \cap \widetilde{\mathbf{B}}^{\dagger, r}$. Let $\mathbf{B}_{\mathrm{rig}, K}^{\dagger, r}$ be the Frechet completion of $\mathbf{B}_{K}^{\dagger, r}$ for the topology induced from that on $\widetilde{\mathbf{B}}_{\text {rig }}^{\dagger, r}$, and put $\mathbf{B}_{\mathrm{rig}, K}^{\dagger}=\cup_{r>0} \mathbf{B}_{\mathrm{rig}, K}^{\dagger, r}$ equipped with the inductive limit topology. Frobunius induces injections $\mathbf{B}_{\mathrm{rig}, K}^{\dagger, r} \hookrightarrow \mathbf{B}_{\mathrm{rig}, K}^{\dagger, p r}$ and $\mathbf{B}_{\mathrm{rig}, K}^{\dagger} \hookrightarrow \mathbf{B}_{\mathrm{rig}, K}^{\dagger}$; there are continuous $\Gamma_{K^{-}}$-actions on $\mathbf{B}_{\text {rig, } K}^{\dagger, r}$ and $\mathbf{B}_{\text {rig }, K}^{\dagger}$.

We end this subsection by the definition of $E-\left(\varphi, \Gamma_{K}\right)$-modules 11$]$. 
Definition 2.1. An $E$ - $\left(\varphi, \Gamma_{K}\right)$-module is a finite $\mathbf{B}_{\text {rig, } K}^{\dagger} \otimes \mathbf{Q}_{p} E$-module $M$ equipped with a Frobenius semilinear action $\varphi_{M}$ and a comtinuous semilinear $\Gamma_{K}$-action such that $M$ is free as a $\mathbf{B}_{\text {rig, } K^{-}}^{\dagger}$-module, that $\operatorname{id}_{\mathbf{B}_{\text {rig, }, K}^{\dagger}} \otimes \varphi_{M}$ : $\mathbf{B}_{\text {rig, }, K}^{\dagger} \otimes_{\varphi, \mathbf{B}_{\text {rig, } K}^{\dagger}} M \rightarrow M$ is an isomorphism, and that $\varphi_{M}$ and the $\Gamma_{K}$-action commute with each other.

By [11, Lemma 1.30] if $M$ is an $E-\left(\varphi, \Gamma_{K}\right)$-module, then $M$ is free over $\mathbf{B}_{\text {rig, }, K}^{\dagger} \otimes \mathbf{Q}_{p} E$.

\subsection{B-pairs}

We recall the theory of $E$ - $B$-pairs [3, 11].

Put $\mathbf{B}_{e, E}=\mathbf{B}_{e} \otimes_{\mathbf{Q}_{p}} E, \mathbf{B}_{\mathrm{dR}, E}^{+}=\mathbf{B}_{\mathrm{dR}}^{+} \otimes_{\mathbf{Q}_{p}} E$ and $\mathbf{B}_{\mathrm{dR}, E}=\mathbf{B}_{\mathrm{dR}} \otimes_{\mathbf{Q}_{p}} E$. We extend the $G_{K}$-actions $E$-linearly to these rings.

Definition 2.2. An $E$-B-pair of $G_{K}$ is a couple $W=\left(W_{e}, W_{\mathrm{dR}}^{+}\right)$such that

- $W_{e}$ is a finite $\mathbf{B}_{e, E}$-module with a continuous semilinear action $G_{K}$-action which is free as a $\mathbf{B}_{e}$-module.

- $W_{\mathrm{dR}}^{+} \subset W_{\mathrm{dR}}=\mathbf{B}_{\mathrm{dR}} \otimes_{\mathbf{B}_{e}} W_{e}$ is a $G_{K}$-stable $\mathbf{B}_{\mathrm{dR}, E^{-}}^{+}$lattice.

By [11, Remark 1.3] $W_{e}$ is free over $\mathbf{B}_{e, E}$ and $W_{\mathrm{dR}}^{+}$is free over $\mathbf{B}_{\mathrm{dR}, E}^{+}$.

If $V$ is an $E$-representation of $G_{K}$, then $W(V)=\left(\mathbf{B}_{e, E} \otimes_{E} V, \mathbf{B}_{\mathrm{dR}, E}^{+} \otimes_{E}\right.$ $V)$ is an $E$-B-pair, called the $E$-B-pair attached to $V$.

If $S$ is a Banach $E$-algebra, we can define $S$ - $B$-pairs similarly; to each $S$-representation $V$ of $G_{K}$ is associated an $S$-B-pair $W(V)=\left(\mathbf{B}_{e, E} \otimes_{E}\right.$ $\left.V, \mathbf{B}_{\mathrm{dR}, E}^{+} \otimes_{E} V\right)$.

If $W_{1}=\left(W_{1, e}, W_{1, \mathrm{dR}}^{+}\right)$and $W_{2}=\left(W_{2, e}, W_{2, \mathrm{dR}}^{+}\right)$are two $E$ - $B$-pairs, we define $W_{1} \otimes W_{2}$ to be

$$
\left(W_{1, e} \bigotimes_{\mathbf{B}_{e, E}} W_{2, e}, W_{1, \mathrm{dR}}^{+} \bigotimes_{\mathbf{B}_{\mathrm{dR}, E}^{+}} W_{2, \mathrm{dR}}^{+}\right)
$$

Here, $W_{1, e} \underset{\mathbf{B}_{e, E}}{\bigotimes} W_{2, e}$ is equipped with the diagonal $G_{K}$-action, and $W_{1, \mathrm{dR}}^{+} \otimes_{\mathbf{B}_{\mathrm{dR}, E}^{+}}$ 
$W_{2, \mathrm{dR}}^{+}$is naturally considered as a $G_{K}$-stable $\mathbf{B}_{\mathrm{dR}, E^{-}}^{+}$lattice of

$$
\mathbf{B}_{\mathrm{dR}} \otimes_{\mathbf{B}_{e}}\left(W_{1, e} \bigotimes_{\mathbf{B}_{e, E}} W_{2, e}\right)=W_{1, \mathrm{dR}} \bigotimes_{\mathbf{B}_{\mathrm{dR}, E}} W_{2, \mathrm{dR}}
$$

where $W_{1, \mathrm{dR}}=\mathbf{B}_{\mathrm{dR}} \otimes_{\mathbf{B}_{e}} W_{1, e}$ and $W_{2, \mathrm{dR}}=\mathbf{B}_{\mathrm{dR}} \otimes_{\mathbf{B}_{e}} W_{2, e}$.

If $W=\left(W_{e}, W_{\mathrm{dR}}^{+}\right)$is an $E$ - $B$-pair with $W_{\mathrm{dR}}=\mathbf{B}_{\mathrm{dR}} \otimes_{\mathbf{B}_{e}} W_{e}$, we define the dual of $W$ to be $W^{*}=\left(W_{e}^{*}, W_{\mathrm{dR}}^{*,+}\right)$, where $W_{e}^{*}$ is $\operatorname{Hom}_{\mathbf{B}_{e}}\left(W, \mathbf{B}_{e}\right)$ equipped with the natural $G_{K^{-}}$action, and $W_{\mathrm{dR}}^{*,+}$ is the $G_{K}$-stable lattice of $\mathbf{B}_{\mathrm{dR}} \otimes_{\mathbf{B}_{e}}$ $W_{e}^{*} \cong \operatorname{Hom}_{\mathbf{B}_{\mathrm{dR}}}\left(W_{\mathrm{dR}}, \mathbf{B}_{\mathrm{dR}}\right)$ defined by

$$
\left\{\ell \in \operatorname{Hom}_{\mathbf{B}_{\mathrm{dR}}}\left(W_{\mathrm{dR}}, \mathbf{B}_{\mathrm{dR}}\right): \ell(x) \in \mathbf{B}_{\mathrm{dR}}^{+} \text {for all } x \in W_{\mathrm{dR}}^{+}\right\} .
$$

The relation between $\left(\varphi, \Gamma_{K}\right)$-modules and $B$-pairs is built by Berger [3]. We recall Berger's construction below.

Let $M$ be a $\left(\varphi, \Gamma_{K}\right)$-module of rank $d$ over the Robba ring $\mathbf{B}_{\text {rig, } K}^{\dagger}$. Berger [3] showed that

$$
W_{e}(M):=\left(\widetilde{\mathbf{B}}_{\text {rig }}^{\dagger}[1 / t] \otimes_{\mathbf{B}_{\text {rig }, K}^{\dagger}} M\right)^{\varphi=1}
$$

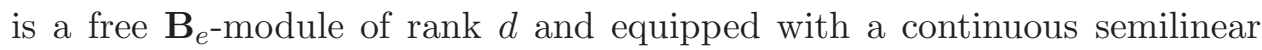
$G_{K}$-action.

For sufficiently large $r_{0}>0$ we can take a unique $\Gamma_{K}$-stable finite free $\mathbf{B}_{\text {rig, } K^{\dagger}}^{\dagger, r}$ submodule $M^{r} \subset M$ such that

$$
\mathbf{B}_{\text {rig, } K}^{\dagger} \otimes_{\mathbf{B}_{\text {rig }, K}^{\dagger, r}} M^{r}=M
$$

and

$$
\operatorname{id}_{\mathbf{B}_{\mathrm{rig}, K}^{\dagger, p r}} \otimes \varphi_{M}: \mathbf{B}_{\mathrm{rig}, K}^{\dagger, p r} \otimes_{\mathbf{B}_{\mathrm{rig}, K}^{\dagger, r}} M^{r} \stackrel{\sim}{\rightarrow} M^{p r}
$$

for any $r \geq r_{0}$. Berger [3] showed that the $\mathbf{B}_{\mathrm{dR}}^{+}$-module

$$
W_{\mathrm{dR}}^{+}(M):=\mathbf{B}_{\mathrm{dR}}^{+} \otimes_{i_{n}, \mathbf{B}_{\mathrm{rig}, K}^{\dagger,(p-1) p^{n-1}}} M^{(p-1) p^{n-1}}
$$

is independent of any $n$ such that $(p-1) p^{n-1} \geq r_{0}$, and showed that there is a canonical $G_{K^{-}}$equivariant isomorphism $\mathbf{B}_{\mathrm{dR}} \otimes_{\mathbf{B}_{e}} W_{e}(M) \stackrel{\sim}{\rightarrow} \mathbf{B}_{\mathrm{dR}} \otimes_{\mathbf{B}_{\mathrm{dR}}^{+}}$ $W_{\mathrm{dR}}^{+}(M)$. 
Put $W(M)=\left(W_{e}(M), W_{\mathrm{dR}}^{+}(M)\right)$. This is an $E$ - $B$-pair of rank $d=$ $\operatorname{rank}_{\mathbf{B}_{\mathrm{rig}, K}^{\dagger} M}$.

The following is a variant version of Berger's result [3, Theorem 2.2.7].

Proposition 2.3 (11], Theorem 1.36). The functor $M \mapsto W(M)$ is an exact functor and this gives an equivalence of categories between the category of $E$ - $\left(\varphi, \Gamma_{K}\right)$-modules and the category of $E$-B-pairs of $G_{K}$.

Proposition 2.4. The functor $M \mapsto W(M)$ respects the tensor products and duals.

Proof. Let $M_{1}$ and $M_{2}$ be two $E-\left(\varphi, \Gamma_{K}\right)$-modules. By taking $\varphi$-invariants, the isomorphism

$$
\begin{aligned}
\left(\widetilde{\mathbf{B}}_{\mathrm{rig}}^{\dagger}[1 / t] \otimes_{\widetilde{\mathbf{B}}_{\mathrm{rig}, K}^{\dagger, r}} M_{1}\right) \otimes_{\widetilde{\mathbf{B}}_{\mathrm{rig}}^{\dagger} \otimes \mathbf{Q}_{p} E[1 / t]}\left(\widetilde{\mathbf{B}}_{\mathrm{rig}}^{\dagger}[1 / t] \otimes_{\widetilde{\mathbf{B}}_{\mathrm{rig}, K}^{\dagger, r}} M_{2}\right) \\
\stackrel{\sim}{\longrightarrow} \widetilde{\mathbf{B}}_{\mathrm{rig}}^{\dagger}[1 / t] \otimes_{\widetilde{\mathbf{B}}_{\mathrm{rig}, K}^{\dagger}}\left(M_{1} \otimes M_{2}\right)
\end{aligned}
$$

induces a $G_{K}$-equivariant injective map

$$
W_{e}\left(M_{1}\right) \otimes \mathbf{B}_{e, E} W_{e}\left(M_{2}\right) \rightarrow W_{e}\left(M_{1} \otimes M_{2}\right) .
$$

Here, $M_{1} \otimes M_{2}$ denotes the $E$ - $\left(\varphi, \Gamma_{K}\right)$-module $M_{1} \otimes_{\mathbf{B}_{\mathrm{rig}, K}^{\dagger} \otimes_{\mathbf{Q}_{p}} E} M_{2}$. Comparing dimensions and using [11, Lemma 1.10] we see that this map is in fact an isomorphism. From the above Berger's construction we see that the natural map

$$
W_{\mathrm{dR}}^{+}\left(M_{1}\right) \otimes_{\mathbf{B}_{\mathrm{dR}}^{+} \otimes_{\mathbf{Q}_{p} E}} W_{\mathrm{dR}}^{+}\left(M_{2}\right) \rightarrow W_{\mathrm{dR}}^{+}\left(M_{1} \otimes M_{2}\right)
$$

is an isomorphism. This proves that the functor $M \mapsto W(M)$ respects tensor products. The proof of that it respects duals is similar.

\subsection{Semistable $E$ - $B$-pairs}

Definition 2.5. An $E-(\varphi, N)$-module over $K$ is a $K_{0} \otimes_{\mathbf{Q}_{p}} E$-module $D$ with a $\varphi \otimes 1$-semilinear isomorphism $\varphi_{D}: D \rightarrow D$, and a $K_{0} \otimes_{\mathbf{Q}_{p}} E$-linear map $N_{D}: D \rightarrow D$ such that $N_{D} \varphi_{D}=p \varphi_{D} N_{D}$. A filtered $E-(\varphi, N)$-module over $K$ is an $E$ - $(\varphi, N)$-module with an exhaustive $\mathbf{Z}$-indexed descending filtration Fil $\bullet^{\bullet}$ on $K \otimes_{K_{0}} D$. 
We have an isomorphism of rings

$$
K \otimes \mathbf{Q}_{p} E \stackrel{\sim}{\rightarrow} \bigoplus_{\tau \in \operatorname{Emb}(K, E)} E_{\tau}, \quad a \otimes b \mapsto(\tau(a) b)_{\tau},
$$

where $E_{\tau}$ is a copy of $E$ for each $\tau \in \operatorname{Emb}(K, E)$. Let $e_{\tau}$ be the unity of $E_{\tau}$. Then $1=\sum_{\tau} e_{\tau}$. Put $D_{\tau}=e_{\tau}\left(K \otimes_{K_{0}} D\right)$. Then $K \otimes_{K_{0}} D=\bigoplus_{\tau \in \operatorname{Emb}(K, E)} D_{\tau}$. Let $\mathrm{Fil}_{\tau}$ denote the induced filtration on $D_{\tau}$.

Definition 2.6. Let $W=\left(W_{e}, W_{\mathrm{dR}}^{+}\right)$be an $E$ - $B$-pair. We define $\mathbf{D}_{\text {cris }}(W)=$ $\left(\mathbf{B}_{\max } \otimes_{\mathbf{B}_{e}} W_{e}\right)^{G_{K}}, \mathbf{D}_{\mathrm{st}}(W)=\left(\mathbf{B}_{\log } \otimes_{\mathbf{B}_{e}} W_{e}\right)^{G_{K}}$ and $\mathbf{D}_{\mathrm{dR}}(W)=\left(\mathbf{B}_{\mathrm{dR}} \otimes_{\mathbf{B}_{e}}\right.$ $\left.W_{e}\right)^{G_{K}}$. Then we have $\operatorname{dim}_{K_{0}}\left(\mathbf{D}_{?}(W)\right) \leq \operatorname{rank}_{\mathbf{B}_{e}} W_{e}$ for ? = cris, st, and $\operatorname{dim}_{K}\left(\mathbf{D}_{\mathrm{dR}}(W)\right) \leq \operatorname{rank}_{\mathbf{B}_{e}} W_{e}$. We say that $W$ is crystalline (resp. semistable) if $\operatorname{dim}_{K_{0}}\left(\mathbf{D}_{?}(W)\right):=\operatorname{rank}_{\mathbf{B}_{e}} W_{e}$ for ? = cris (resp. st).

If $W$ is a semistable $E$ - $B$-pair, we attach to $W$ a filtered $E$ - $(\varphi, N)$ module as follows. The underlying $E-(\varphi, N)$-module is $\mathbf{D}_{\text {st }}(W)$; the filtration on $\mathbf{D}_{\mathrm{dR}}(W)=K \otimes_{K_{0}} \mathbf{D}_{\mathrm{st}}(W)$ is given by $\operatorname{Fil}^{i} \mathbf{D}_{\mathrm{dR}}(W)=t^{i} W_{\mathrm{dR}}^{+} \cap \mathbf{D}_{\mathrm{dR}}(W)$.

\section{Proposition 2.7.}

(a) The functor $W \mapsto \mathbf{D}_{\text {st }}(W)$ realizes an equivalence of categories between the category of semistable $E$-B-pairs of $G_{K}$ and the category of filtered $E-(\varphi, N)$-modules over $K$.

(b) If $W_{1}$ and $W_{2}$ are semistable, then so is $W_{1} \otimes W_{2}$.

(c) The functor $W \mapsto \mathbf{D}_{\mathrm{st}}(W)$ respects the tensor products and duals.

(d) If

$$
0 \longrightarrow W_{1} \longrightarrow W \longrightarrow W_{2} \longrightarrow 0
$$

is a short exact sequence of E-B-pairs, and $W$ is semistable, then $W_{1}$ and $W_{2}$ are semistable.

(e) The functor $W \mapsto \mathbf{D}_{\text {st }}(W)$ is exact.

Proof. Assertion (国) follows from [3, Proposition 2.3.4]. See also [11, Theorem $1.18(2)]$.

Let $W_{1}$ and $W_{2}$ be two $E$ - $B$-pairs. The isomorphism

$$
\left(\mathbf{B}_{\log } \otimes_{\mathbf{B}_{e}} W_{1}\right) \otimes_{\mathbf{B}_{\log } \otimes_{\mathbf{Q}_{p}} E}\left(\mathbf{B}_{\log } \otimes_{\mathbf{B}_{e}} W_{2}\right) \stackrel{\sim}{\rightarrow} \mathbf{B}_{\log } \otimes_{\mathbf{B}_{e}}\left(W_{1} \otimes W_{2}\right)
$$


induces an injective map

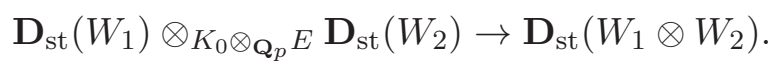

When $W_{1}$ and $W_{2}$ are semistable, the dimension of the source over $K_{0}$ is

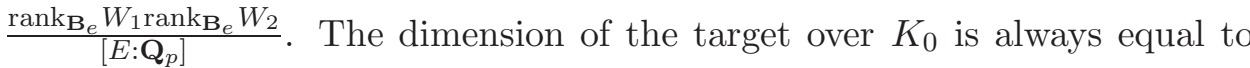
or less than $\operatorname{rank}_{\mathbf{B}_{e}}\left(W_{1} \otimes W_{2}\right)=\frac{\operatorname{rank}_{\mathbf{B}_{e}} W_{1} \operatorname{rank}_{\mathbf{B}_{e}} W_{2}}{\left[E: \mathbf{Q}_{p}\right]}$. Hence, (2.2) is an isomorphism, and so $W_{1} \otimes W_{2}$ is semistable. This proves (b). Similarly, the isomorphism

$$
\left(\mathbf{B}_{\mathrm{dR}} \otimes_{\mathbf{B}_{e}} W_{1}\right) \otimes_{\mathbf{B}_{\mathrm{dR}} \otimes_{\mathbf{Q}_{p}} E}\left(\mathbf{B}_{\mathrm{dR}} \otimes_{\mathbf{B}_{e}} W_{2}\right) \stackrel{\sim}{\rightarrow} \mathbf{B}_{\mathrm{dR}} \otimes_{\mathbf{B}_{e}}\left(W_{1} \otimes W_{2}\right)
$$

induces an isomorphism

$$
\mathbf{D}_{\mathrm{dR}}\left(W_{1}\right) \otimes_{K \otimes_{\mathbf{Q}_{p}} E} \mathbf{D}_{\mathrm{dR}}\left(W_{2}\right) \rightarrow \mathbf{D}_{\mathrm{dR}}\left(W_{1} \otimes W_{2}\right)
$$

Via the isomorphism (2.3) the filtration on $\left(\mathbf{B}_{\mathrm{dR}} \otimes_{\mathbf{B}_{e}} W_{1}\right) \otimes_{\mathbf{B}_{\mathrm{dR}} \otimes \mathbf{Q}_{p} E}\left(\mathbf{B}_{\mathrm{dR}} \otimes_{\mathbf{B}_{e}}\right.$ $\left.W_{2}\right)$ coincides with that on $\mathbf{B}_{\mathrm{dR}} \otimes_{\mathbf{B}_{e}}\left(W_{1} \otimes W_{2}\right)$. Therefore, the filtration on $\mathbf{D}_{\mathrm{dR}}\left(W_{1}\right) \otimes_{K \otimes \mathbf{Q}_{p} E} \mathbf{D}_{\mathrm{dR}}\left(W_{2}\right)$ and that on $\mathbf{D}_{\mathrm{dR}}\left(W_{1} \otimes W_{2}\right)$ coincide. Indeed, they are the restrictions of the filtrations on $\left(\mathbf{B}_{\mathrm{dR}} \otimes_{\mathbf{B}_{e}} W_{1}\right) \otimes_{\mathbf{B}_{\mathrm{dR}} \otimes_{\mathbf{Q}_{p}} E}$ $\left(\mathbf{B}_{\mathrm{dR}} \otimes_{\mathbf{B}_{e}} W_{2}\right)$ and $\mathbf{B}_{\mathrm{dR}} \otimes_{\mathbf{B}_{e}}\left(W_{1} \otimes W_{2}\right)$ respectively. Similarly we can show that $W \mapsto \mathbf{D}_{\mathrm{st}}(W)$ respects duals. This proves (C).

For (d) we have the following exact sequence

$$
0 \longrightarrow \mathbf{D}_{\text {st }}\left(W_{1}\right) \longrightarrow \mathbf{D}_{\text {st }}(W) \longrightarrow \mathbf{D}_{\text {st }}\left(W_{2}\right)
$$

So (d) follows from a dimension argument. Furthermore, when $W$ is semistable, $\mathbf{D}_{\text {st }}(W) \rightarrow \mathbf{D}_{\text {st }}\left(W_{2}\right)$ is surjective. For any $i \in \mathbf{Z}$ we write $d_{i}(W)$ for $\operatorname{dim}_{K} \mathrm{Fil}^{i} \mathbf{D}_{\text {st }}(W)$. As the maps in the exact sequence (2.4) respect filtrations, we have $d_{i}(W) \leq d_{i}\left(W_{1}\right)+d_{i}\left(W_{2}\right)$. Similarly, we have $d_{1-i}\left(W^{*}\right) \leq$ $d_{1-i}\left(W_{1}^{*}\right)+d_{1-i}\left(W_{2}^{*}\right)$. As $W \mapsto \mathbf{D}_{\text {st }}(W)$ respects duals, we have $d_{i}(W)=$ $\operatorname{dim}_{K}\left(\mathbf{D}_{\mathrm{dR}}(W)\right)-d_{1-i}\left(W^{*}\right)$. Then

$$
\begin{aligned}
d_{i}(W) & =\operatorname{dim}_{K}\left(\mathbf{D}_{\mathrm{dR}}(W)\right)-d_{1-i}\left(W^{*}\right) \\
& \geq\left(\operatorname{dim}_{K}\left(\mathbf{D}_{\mathrm{dR}}\left(W_{1}\right)\right)-d_{1-i}\left(W_{1}^{*}\right)\right)+\operatorname{dim}_{K}\left(\mathbf{D}_{\mathrm{dR}}\left(W_{2}\right)\right)-d_{1-i}\left(W_{2}^{*}\right) \\
& =d_{i}\left(W_{1}\right)+d_{i}\left(W_{2}\right) .
\end{aligned}
$$


Thus we must have $d_{i}(W)=d_{i}\left(W_{1}\right)+d_{i}\left(W_{2}\right)$ for all $i \in \mathbf{Z}$. In other words, the maps in (2.4) are strict for the filtrations, which shows (国).

By [3, Proposition 2.3.4] the quasi-inverse of the functor $\mathbf{D}_{\text {st }}$ is given by

$$
\mathbf{D}_{B}(D)=\left(\left(\mathbf{B}_{\log } \otimes_{K_{0}} D\right)^{\varphi=1, N=0}, \mathrm{Fil}^{0}\left(\mathbf{B}_{\mathrm{dR}} \otimes_{K_{0}} D\right)\right)
$$

For a filtered $E-(\varphi, N)$-module $D$ we put

$\mathbf{X}_{\log }(D)=\left(\mathbf{B}_{\log } \otimes_{K_{0}} D\right)^{\varphi=1, N=0}$ and $\mathbf{X}_{\mathrm{dR}}(D)=\mathbf{B}_{\mathrm{dR}} \otimes_{K_{0}} D / \mathrm{Fil}^{0}\left(\mathbf{B}_{\mathrm{dR}} \otimes_{K_{0}} D\right)$

If $\mathbf{D}_{B}(D)=\left(W_{e}, W_{\mathrm{dR}}^{+}\right)$, then $\mathbf{X}_{\log }(D)=W_{e}$ and $\mathbf{X}_{\mathrm{dR}}(D)=\left(\mathbf{B}_{\mathrm{dR}} \otimes_{\mathbf{B}_{e}}\right.$ $\left.W_{e}\right) / W_{\mathrm{dR}}^{+}$.

\section{3. $S$-B-pairs of Rank 1 and Triangulations}

\section{1. $S$ - $B$-pairs of rank 1}

Let $S$ be a Banach $E$-algebra.

For any $a \in S^{\times}$we define a filtered $S$ - $\varphi$-module $D_{a}$ as follows. As a $K_{0} \otimes_{\mathbf{Q}_{p}} S$-module,

$$
D_{a}=K_{0} \otimes_{\mathbf{Q}_{p}} S=\oplus_{\tau: K_{0} \hookrightarrow E} S e_{\tau}
$$

the $\varphi \otimes 1$-semilinear action $\varphi$ on $D_{a}$ satisfies

$$
\varphi\left(e_{\mathrm{id}}\right)=e_{\varphi^{-1}}, \varphi\left(e_{\varphi^{-1}}\right)=e_{\varphi^{-2}}, \ldots, \varphi\left(e_{\varphi^{1-f}}\right)=a e_{\mathrm{id}}
$$

the descending filtration on $D_{a, K}=K \otimes_{\mathbf{Q}_{p}} S$ is given by $\mathrm{Fil}^{0} D_{a, K}=D_{a, K}$ and $\mathrm{Fil}^{1} D_{a, K}=0$.

Lemma 3.1. If $a \in S$ satisfies that $a-1$ is topologically nilpotent, then there exists a unit $u_{0} \in \mathbf{B}_{\max } \widehat{\otimes}_{K_{0}} S$ such that $\varphi^{\left[K_{0}: \mathbf{Q}_{p}\right]}\left(u_{0}\right)=a u_{0}$. Consequently

$$
\left\{x \in \mathbf{B}_{\max } \widehat{\otimes}_{K_{0}} S: \varphi^{\left[K_{0}: \mathbf{Q}_{p}\right]}(x)=a x\right\}=\left(\mathbf{B}_{e, K_{0}} \widehat{\otimes}_{K_{0}} S\right) u_{0} .
$$

Proof. Let $\mathbf{Q}_{p}^{\text {ur }}$ be the completed unramified extension of $\mathbf{Q}_{p}$. Then there exists an inclusion $\mathbf{Q}_{p}^{\mathrm{ur}} \hookrightarrow \mathbf{B}_{\max }$ that is compatible with $\varphi$. 
As $\varphi^{\left[K_{0}: \mathbf{Q}_{p}\right]}-1$ is surjective on $\mathbf{Q}_{p}^{\mathrm{ur}}$, there exists a sequence $c_{0}=1, c_{1}, \cdots$ of elements in $\mathbf{Q}_{p}^{\text {ur }}$ such that

$$
\left(\varphi^{\left[K_{0}: \mathbf{Q}_{p}\right]}-1\right) c_{i}=c_{i-1}
$$

for $i \geq 1$. The image of $c_{i}$ by the map

$$
\mathbf{Q}_{p}^{\mathrm{ur}} \hookrightarrow \mathbf{B}_{\max } \rightarrow \mathbf{B}_{\max } \widehat{\otimes}_{K_{0}} S
$$

is again denoted by $c_{i}$. Put

$$
u_{0}=\sum_{i=0}^{\infty} c_{i}(a-1)^{i}
$$

Then $u_{0}$ is a unit and we have $\varphi^{\left[K_{0}: \mathbf{Q}_{p}\right]} u_{0}=a u_{0}$.

Proposition 3.2. If $a \in S$ satisfies that $a-1$ is topologically nilpotent, then $\mathbf{D}_{B}\left(D_{a}\right)$ is an $S$-B-pair of rank 1 . Here $\mathbf{D}_{B}$ is the functor defined by (2.5).

Proof. For each $z \in \mathbf{B}_{\max } \widehat{\otimes}_{\mathbf{Q}_{p}} D_{a}$ we write $z=\sum c_{\tau} e_{\tau}$ with $c_{\tau} \in \mathbf{B}_{\max } \widehat{\otimes}_{K_{0}, \tau} S$. Then $\varphi(z)=z$ if and only if $\varphi\left(c_{\varphi^{i}}\right)=c_{\varphi^{i-1}}\left(i=1, \ldots,\left[K_{0}: \mathbf{Q}_{p}\right]\right)$ and $\varphi^{\left[K_{0}: \mathbf{Q}_{p}\right]}\left(c_{\mathrm{id}}\right)=a c_{\mathrm{id}}$. Our assertion follows from Lemma 3.1.

For any $a \in S^{\times}$, let $\delta_{a}: K^{\times} \rightarrow S^{\times}$denote the character such that $\delta_{a}\left(\pi_{K}\right)=a$ and $\left.\delta_{a}\right|_{\mathfrak{o}_{K}^{\times}}=1$.

Remark 3.3. In the case of $S=E$, for any $u \in E^{\times}, \mathbf{D}_{B}\left(D_{u}\right)$ coincides with the $E$ - $B$-pair $W\left(\delta_{u}\right)$ defined in [11] (see [11, §1.4]). From now on the base change of $W\left(\delta_{u}\right)$ from $E$ to $S$ is again denoted by $W\left(\delta_{u}\right)$.

Let $\delta: K^{\times} \rightarrow S^{\times}$be a continuous character such that $\delta\left(\pi_{K}\right)$ is of the form $\delta\left(\pi_{K}\right)=a u$, where $u \in E^{\times}$and $a \in S$ satisfies that $a-1$ is topologically nilpotent. We call such a character a good character. Let $W_{a}$ be the resulting $S$ - $B$-pair in Proposition 3.2. Let $\delta^{\prime}$ be the unitary continuous character $K^{\times} \rightarrow E^{\times}$such that $\left.\delta^{\prime}\right|_{\mathfrak{o}_{K}^{\times}}=\left.\delta\right|_{\mathfrak{o}_{K}^{\times}}$and $\delta^{\prime}\left(\pi_{K}\right)=1$. By local class field theory, this induces a continuous character $\widetilde{\delta}^{\prime}: G_{K} \rightarrow S^{\times}$such that $\widetilde{\delta}^{\prime} \circ \operatorname{rec}_{K}=\delta^{\prime}$. Then we put

$$
W(\delta)=W\left(S\left(\widetilde{\delta}^{\prime}\right)\right) \otimes W\left(\delta_{u}\right) \otimes W_{a},
$$

where $W\left(S\left(\widetilde{\delta^{\prime}}\right)\right)$ is the $S$-B-pair attached to the Galois representation $S\left(\widetilde{\delta^{\prime}}\right)$. 
If $\delta$ is a continuous character $\delta: K^{\times} \rightarrow S^{\times}$, we write $\log (\delta)$ for the logarithmic of $\left.\delta\right|_{\mathfrak{o}_{K}^{\times}}$, which is a $\mathbf{Z}_{p}$-linear homomorphism $\log (\delta): K \rightarrow S$.

For any $\tau \in \operatorname{Emb}(K, E)$ we use the same notation $\tau$ to denote the composition of $\tau: K \hookrightarrow E$ and $E \hookrightarrow S$. Then $\{\tau: K \hookrightarrow S\}$ is a basis of $\operatorname{Hom}_{\mathbf{z}_{p}}(E, S)$ over $S$. Write $\log (\delta)=\sum_{\tau} k_{\tau} \tau, k_{\tau} \in S$. We call $\left(k_{\tau}\right)_{\tau}$ the weight vector of $\delta$ and denote it by $\vec{w}(\delta)$. We use $w_{\tau}(\delta)$ to denote $k_{\tau}$.

Remark 3.4. Let $S$ be an affinoid algebra over $E$. For any continuous character $\delta: K^{\times} \rightarrow S^{\times}$and any point $z_{0}$ of $\operatorname{Max}(S)$, there exists an affinoid neighborhood $U=\operatorname{Max}\left(S^{\prime}\right)$ of $z_{0}$ in $\operatorname{Max}(S)$ such that the restriction of $\delta$ to $U$ is good.

Lemma 3.5. Let $\delta$ be a character of $K^{\times}$with values in $S=E[Z] /\left(Z^{2}\right), \bar{\delta}$ the character of $K^{\times}$with values in $E$ obtained from $\delta$ modulo $(Z)$. Write $\delta=\bar{\delta}_{S}(1+Z \epsilon)$, where $\bar{\delta}_{S}$ is the character $K^{\times} \stackrel{\bar{\delta}}{\rightarrow} E^{\times} \hookrightarrow S^{\times}$. Let $\epsilon^{\prime}$ be the additive character of $G_{K}$ such that $\epsilon^{\prime} \circ \operatorname{rec}_{K}(p)=0$ and $\left.\epsilon^{\prime} \circ \operatorname{rec}_{K}\right|_{\mathfrak{o}_{K}^{\times}}=\left.\epsilon\right|_{\mathfrak{o}_{K}^{\times}}$.

Assume that $W(\bar{\delta})$ is crystalline and $\varphi^{\left[K_{0}: \mathbf{Q}_{p}\right]}$ acts on $\mathbf{D}_{\text {cris }}(W(\bar{\delta}))$ by $\alpha$. Then there is a nonzero element

$$
x \in\left(\mathbf{B}_{\max , E} \otimes_{\mathbf{B}_{e, E}} W(\delta)_{e}\right)^{\varphi^{\left[K_{0}: \mathbf{Q}_{p}\right]}=\alpha\left(1+Z v_{p}\left(\pi_{K}\right) \epsilon(p)\right), G_{K}=\left(1+Z \epsilon^{\prime}\right)}
$$

whose reduction modulo $Z$ is a basis of $\mathbf{D}_{\mathrm{st}}(W(\bar{\delta}))$ over $K \otimes_{\mathbf{Q}_{p}} E$.

Proof. This follows from the fact that $W(\delta)=W\left(\bar{\delta}_{S}\right) \otimes W_{\delta_{1+Z v_{p}\left(\pi_{K}\right) \epsilon(p)}} \otimes$ $W\left(1+Z \epsilon^{\prime}\right)$.

\subsection{Triangulations and refinements}

Now let $S$ be an affinoid algebra over $E$. For any open affinoid subset $U$ of $S$ and any $S$-B-pair $W$ let $W_{U}$ denote the restriction to $U$ of $W$.

Definition 3.6. Let $W$ be an $S$ - $B$-pair of $\operatorname{rank} n, z_{0}$ a point of $\operatorname{Max}(S)$. If there is

- an affinoid neighborhood $U=\operatorname{Max}\left(S_{U}\right)$ of $z_{0}$,

- a strictly increasing filtration

$$
\{0\}=\mathrm{Fil}_{0} W_{U} \subset \mathrm{Fil}_{1} W_{U} \subset \cdots \subset \mathrm{Fil}_{n} W_{U}=W_{U}
$$


of saturated free sub- $S_{U}-B$-pairs, and

- $n$ good continuous characters $\delta_{i}: \mathbf{Q}_{p}^{\times} \rightarrow S_{U}^{\times}$

such that for any $i=1, \ldots, n$,

$$
\operatorname{Fil}_{i} W_{U} / \mathrm{Fil}_{i-1} W_{U} \simeq W\left(\delta_{i}\right),
$$

we say that $W$ is locally triangulable at $z_{0}$; we call Fil• a local triangulation of $W$ at $z_{0}$, and call $\left(\delta_{1}, \ldots, \delta_{n}\right)$ the local triangulation parameters attached to Fil.

Please consult [6, 4] for more knowledge on triangulations.

To discuss the relation between triangulations and refinements, we restrict ourselves to the case of $S=E$.

Let $D$ be a filtered $E$ - $(\varphi, N)$-module of rank $n$. The operator $\varphi^{\left[K_{0}: \mathbf{Q}_{p}\right]}$ on $D$ is $K_{0} \otimes \mathbf{Q}_{p} E$-linear. We assume that the eigenvalues of $\varphi^{\left[K_{0}: \mathbf{Q}_{p}\right]}: D \rightarrow D$ are all in $K_{0} \otimes_{\mathbf{Q}_{p}} E$, i.e. there exists a basis of $D$ over $K_{0} \otimes_{\mathbf{Q}_{p}} E$ such the matrix of $\varphi^{\left[K_{0}: \mathbf{Q}_{p}\right]}$ with respect to this basis is upper-triangular.

Following Mazur [9] we define a refinement of $D$ to be a filtration on $D$

$$
0=\mathcal{F}_{0} D \subset \mathcal{F}_{1} D \subset \cdots \subset \mathcal{F}_{n} D=D
$$

by $E$-subspaces stable by $\varphi_{D}$ and $N_{D}$, such that each factor $\operatorname{gr}_{i}^{\mathcal{F}} D=\mathcal{F}_{i} D / \mathcal{F}_{i-1} D(i=1, \ldots, n)$ is of rank 1 over $K_{0} \otimes_{\mathbf{Q}_{p}} E$. Any refinement fixes an ordering $\alpha_{1}, \ldots, \alpha_{n}$ of eigenvalues of $\varphi^{\left[K_{0}: \mathbf{Q}_{p}\right]}$ and an ordering $\vec{k}_{1}, \ldots, \vec{k}_{n}$ of Hodge-Tate weights of $K \otimes_{K_{0}} D$ taken with multiplicities such that the eigenvalue of $\varphi^{\left[K_{0}: \mathbf{Q}_{p}\right]}$ on $\operatorname{gr}_{i}^{\mathcal{F}} D$ is $\alpha_{i}$ and the Hodge-Tate weight of $\operatorname{gr}_{i}^{\mathcal{F}} \mathcal{D}$ is $\vec{k}_{i}$.

We have the following analogue of [1, Proposition 1.3.2].

Proposition 3.7. Let $W$ be a semistable $E$-B-pair, $D=\mathbf{D}_{\mathrm{st}}(W)$.

(a) The equivalence of categories between the category of semistable $E$ - $B$ pairs and the category of filtered $E-(\varphi, N)$-modules induces a bijection between the set of triangulations on $W$ and the set of refinements on $D$. 
(b) If $\left(\mathrm{Fil}_{i} W\right)$ is a triangulation of $W$ with triangulation parameters $\left(\delta_{1}, \ldots, \delta_{n}\right)$ that correspond to a refinement $\mathcal{F}_{\bullet} D$ of $D$ with the ordering of Hodge-Tate weights being $\vec{k}_{1}, \ldots, \vec{k}_{n}$, then $\delta_{i}=\tilde{\delta}_{i} \prod_{\tau \in \operatorname{Emb}(K, E)} \tau(x)^{k_{i, \tau}}$, where $\tilde{\delta}_{i}$ is a smooth character.

Proof. Assertion (国) follows from the fact that $\mathbf{D}_{\text {st }}$ is an exact. Assertion (b) follows from [11, Lemma 4.1].

\section{Cohomology Theory}

\subsection{Cohomology of $\left(\varphi, \Gamma_{K}\right)$-modules and cohomology of $B$-pairs}

Let $M$ be a $\left(\varphi, \Gamma_{K}\right)$-module. Assume that $\Gamma_{K}$ has a topological generator $\gamma$. Define the cohomology $H_{\Phi \Gamma}^{\bullet}(M)$ by the complex $C^{\bullet}(M)$ defined by

$$
C^{0}(M)=M \stackrel{(\gamma-1, \varphi-1)}{\longrightarrow} C^{1}(M)=M \oplus M \rightarrow C^{2}(M)=M,
$$

where the map $C^{1}(M) \rightarrow C^{2}(M)$ is given by $(x, y) \mapsto(\varphi-1) x-(\gamma-1) y$. Denote the kernel of $C^{1}(M) \rightarrow C^{2}(M)$ by $Z^{1}(M)$.

There is a one-to-one correspondence between $H^{1}(M)$ and the set of extensions of $M_{0}$ by $M$ in the category of $\left(\varphi, \Gamma_{K}\right)$-modules, where $M_{0}=$ $\mathbf{B}_{\text {rig, } K}^{\dagger} e_{0}$ is the trivial $\left(\varphi, \Gamma_{K}\right)$-module with $\varphi\left(e_{0}\right)=\gamma\left(e_{0}\right)=e_{0}$. Let $\tilde{M}$ be an extension of $M_{0}$ by $M$, and let $\tilde{e}$ be any lifting of $e_{0}$ in $\tilde{M}$. Then the element in $H^{1}(M)$ corresponding to the extension $\tilde{M}$ is the class of $((\gamma-1) \tilde{e},(\varphi-1) \tilde{e}) \in Z^{1}(M)$.

In 11] Nakamura introduced a cohomology for $B$-pairs and use it to compute the cohomology of $\left(\varphi, \Gamma_{K}\right)$-modules.

If $W=\left(W_{e}, W_{\mathrm{dR}}^{+}\right)$is an $E$ - $B$-pair, let $C^{\bullet}(W)$ be the complex of $G_{K^{-}}$ modules defined by

$$
C^{0}(W):=W_{e} \rightarrow C^{1}(W):=W_{\mathrm{dR}} / W_{\mathrm{dR}}^{+} .
$$

Here, $W_{e} \rightarrow W_{\mathrm{dR}} / W_{\mathrm{dR}}^{+}$is the natural map.

Definition 4.1. Let $W=\left(W_{e}, W_{\mathrm{dR}}^{+}\right)$be an $E$-B-pair. We define the Galois cohomology of $W$ by $H_{B}^{i}(W):=H^{i}\left(G_{K}, C^{\bullet}(W)\right)$. 
By definition there is a long exact sequence

$$
\cdots \rightarrow H_{B}^{i}(W) \rightarrow H^{i}\left(G_{K}, W_{e}\right) \rightarrow H^{i}\left(G_{K}, W_{\mathrm{dR}} / W_{\mathrm{dR}}^{+}\right) \rightarrow \cdots
$$

For a $G_{K}$-module $M$ put $C^{0}(M)=M$ and let $C^{i}(M)$ be the space of continuous functions from $\left(G_{K}\right)^{\times i}$ to $M$. Let $\delta_{0}: C^{0}(M) \rightarrow C^{1}(M)$ be the map $x \mapsto(g \mapsto g(x)-x)$ and let $\delta_{1}: C^{1}(M) \rightarrow C^{2}(M)$ be the map $f \mapsto\left(\left(g_{1}, g_{2}\right) \mapsto f\left(g_{1} g_{2}\right)-f\left(g_{1}\right)-g_{1} f\left(g_{2}\right)\right)$.

Nakamura [11] showed that $H_{B}^{1}(W)$ is isomorphic to $\operatorname{ker}\left(\tilde{\delta}_{1}\right) / \operatorname{im}\left(\tilde{\delta}_{0}\right)$, where $\tilde{\delta}_{0}$ and $\tilde{\delta}_{1}$ are defined by

$$
\begin{aligned}
\tilde{\delta}_{0}: C^{0}\left(W_{e}\right) \oplus C^{0}\left(W_{\mathrm{dR}}^{+}\right) & \rightarrow C^{1}\left(W_{e}\right) \oplus C^{1}\left(W_{\mathrm{dR}}^{+}\right) \oplus C^{0}\left(W_{\mathrm{dR}}\right): \\
(x, y) & \mapsto\left(\delta_{0}(x), \delta_{0}(y), x-y\right), \\
\tilde{\delta}_{1}: C^{1}\left(W_{e}\right) \oplus C^{1}\left(W_{\mathrm{dR}}^{+}\right) \oplus C^{0}\left(W_{\mathrm{dR}}\right) & \rightarrow C^{2}\left(W_{e}\right) \oplus C^{2}\left(W_{\mathrm{dR}}^{+}\right) \oplus C^{1}\left(W_{\mathrm{dR}}\right): \\
\left(f_{1}, f_{2}, x\right) & \mapsto\left(\delta_{1}\left(f_{1}\right), \delta_{1}\left(f_{2}\right), f_{1}-f_{2}-\delta_{0}(x)\right) .
\end{aligned}
$$

The map $H_{B}^{1}(W) \rightarrow H^{1}\left(G_{K}, W_{e}\right)$ is induced by the forgetful map

$$
C^{1}\left(W_{e}\right) \oplus C^{1}\left(W_{\mathrm{dR}}^{+}\right) \oplus C^{0}\left(W_{\mathrm{dR}}\right) \rightarrow C^{1}\left(W_{e}\right) .
$$

There is a one-to-one correspondence between $H^{1}\left(G_{K}, W\right)$ and the set of extensions of $W_{0}$ by $W$ in the category of $E$ - $B$-pairs. Here, $W_{0}=\left(\mathbf{B}_{e} \otimes \mathbf{Q}_{p}\right.$ $\left.E, \mathbf{B}_{\mathrm{dR}}^{+} \otimes_{\mathbf{Q}_{p}} E\right)$ is the trivial $E$ - $B$-pair. Let $\tilde{W}=\left(\tilde{W}_{e}, \tilde{W}_{\mathrm{dR}}^{+}\right)$be an extension of $W_{0}$ by $W$. Let $\left(\tilde{w}_{e}, \tilde{w}_{\mathrm{dR}}^{+}\right)$be a lifting in $\tilde{W}$ of $(1,1) \in W_{0}$. Then the element in $H_{B}^{1}(W)$ corresponding to the extension $\tilde{W}$ is just the class of $\left(\left(\sigma \mapsto(\sigma-1) \tilde{w}_{e}\right),\left(\sigma \mapsto(\sigma-1) \tilde{w}_{\mathrm{dR}}^{+}\right), \tilde{w}_{e}-\tilde{w}_{\mathrm{dR}}^{+}\right) \in \operatorname{ker}\left(\tilde{\delta}_{1}\right)$.

By Proposition 2.3 there is a one-to-one correspondence between $\operatorname{Ext}\left(M_{0}, M\right)$ and

$\operatorname{Ext}\left(W_{0}, W(M)\right)$. It induces a natrual isomorphism

$$
i_{M}: H_{\Phi \Gamma}^{1}(M) \rightarrow H_{B}^{1}(W(M))
$$

\subsection{1-cocycles from infinitesimal deformations}

Let $S$ be the $E$-algebra $E[Z] /\left(Z^{2}\right), \tilde{M}$ an $S$ - $\left(\varphi, \Gamma_{K}\right)$-module. Let $\left\{e_{1}, \ldots\right.$, $\left.e_{n}\right\}$ be an $S$-basis of $\tilde{M},\left\{e_{1}^{*}, \ldots, e_{n}^{*}\right\}$ the dual basis of $\tilde{M}^{*}$. Put $M=\tilde{M} \otimes_{S} E$ 
and $M^{*}=\tilde{M}^{*} \otimes_{S} E$. Let $e_{i, z}$ denote $e_{i} \bmod Z$, and $e_{j, z}^{*}$ denote $e_{j}^{*} \bmod Z$. Then $\left\{e_{1, z}, \ldots, e_{n, z}\right\}$ is an $E$-basis of $M$, and $\left\{e_{1, z}^{*}, \ldots, e_{n, z}^{*}\right\}$ is an $E$-basis of $M^{*}$.

The matrices of $\varphi$ and $\gamma$ with respect to $\left\{e_{1}, \ldots, e_{n}\right\}$ are denote by $\tilde{A}_{\varphi}$ and $\tilde{A}_{\gamma}$ respectively, so that $\varphi\left(e_{j}\right)=\sum_{i}\left(\tilde{A}_{\varphi}\right)_{i j} e_{i}$ and $\gamma\left(e_{j}\right)=\sum_{i}\left(\tilde{A}_{\gamma}\right)_{i j} e_{i}$. Write $\tilde{A}_{\varphi}=\left(I_{n}+Z U_{\varphi}\right) A_{\varphi}$ and $\tilde{A}_{\gamma}=\left(I_{n}+Z U_{\gamma}\right) A_{\gamma}$. Put

$$
c_{\Phi \Gamma}(\tilde{M})=\left(\sum_{i, j}\left(U_{\varphi}\right)_{i, j} e_{j, z}^{*} \otimes e_{i, z}, \sum_{i, j}\left(U_{\gamma}\right)_{i, j} e_{j, z}^{*} \otimes e_{i, z}\right) .
$$

Write $\mathbf{D}_{B}(\tilde{M})=\left(\tilde{W}_{e}, \tilde{W}_{\mathrm{dR}}^{+}\right), \mathbf{D}_{B}(M)=W$ and $\mathbf{D}_{B}\left(M^{*}\right)=W^{*}$.

Let $f_{1}, \ldots, f_{n}$ be a basis of $\tilde{W}_{e}$ over $\mathbf{B}_{e, E}$, and let $g_{1}, \ldots, g_{n}$ be a basis of $\tilde{W}_{\mathrm{dR}}^{+}$over $\mathbf{B}_{\mathrm{dR}, E}^{+}$. We write the matrix of $\sigma \in G_{K}$ with respect to the basis $\left\{f_{1}, \ldots, f_{n}\right\}$ by $\left(I_{n}+Z U_{e, \sigma}\right) A_{e, \sigma}$, and the matrix of $\sigma$ with respect to the basis $\left\{g_{1}, \ldots, g_{n}\right\}$ by $\left(I_{n}+Z U_{\mathrm{dR}, \sigma}^{+}\right) A_{\mathrm{dR}, \sigma}^{+}$. Here,

$$
U_{e, \sigma} \in \mathrm{M}_{n}\left(\mathbf{B}_{e, E}\right), U_{\mathrm{dR}, \sigma}^{+} \in \mathrm{M}_{n}\left(\mathbf{B}_{\mathrm{dR}, E}^{+}\right), A_{e, \sigma} \in \mathrm{GL}_{n}\left(\mathbf{B}_{e, E}\right)
$$

and

$$
A_{\mathrm{dR}, \sigma}^{+} \in \mathrm{GL}_{n}\left(\mathbf{B}_{\mathrm{dR}, E}^{+}\right) .
$$

Write $\left(f_{1}, \ldots, f_{n}\right)=\left(g_{1}, \ldots, g_{n}\right)\left(I_{n}+Z U_{\mathrm{dR}}\right) A_{\mathrm{dR}}$ and put $c_{B}(\tilde{M})=\left(\left(\sigma \mapsto \sum_{i, j}\left(U_{e, \sigma}\right)_{i j} f_{j, z}^{*} \otimes f_{i, z}\right),\left(\sigma \mapsto \sum_{i, j}\left(U_{\mathrm{dR}, \sigma}^{+}\right)_{i j} g_{j, z}^{*} \otimes g_{i, z}\right), \sum_{i, j}\left(U_{\mathrm{dR}}\right)_{i j} g_{j, z}^{*} \otimes g_{i, z}\right)$.

\section{Proposition 4.2.}

(a) $c_{\Phi \Gamma}(\tilde{M})$ is in $Z^{1}\left(M^{*} \otimes M\right)$.

(b) $c_{B}(\tilde{M})$ is in $\operatorname{ker}\left(\tilde{\delta}_{1, W^{*} \otimes W}\right)$.

(c) We have $i_{M}\left(\left[c_{\Phi \Gamma}(\tilde{M})\right]\right)=\left[c_{B}(\tilde{M})\right]$.

Proof. It is easy to verify (国) and (b).

Put $M_{S}^{*}=M^{*} \otimes_{E} S$. We consider $M_{S}^{*} \otimes_{S} \tilde{M}$ as an extension of $M^{*} \otimes_{E} M$ by itself, and form the following commutative diagram

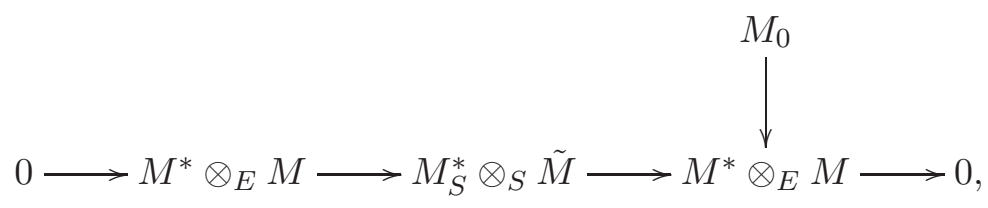


where the vertical map $M_{0} \rightarrow M^{*} \otimes_{E} M$ is given by $1 \mapsto \sum_{i=1}^{n} e_{i, z}^{*} \otimes e_{i, z}$, which does not depend on the choice of the basis $\left\{e_{1}, \ldots, e_{n}\right\}$. Pulling back $M_{S}^{*} \otimes_{S} \tilde{M}$ via $M_{0} \rightarrow M^{*} \otimes_{E} M$ we obtain an extension of $M_{0}$ by $M^{*} \otimes_{E} M$. Let $\mathcal{M}$ denote the resulting extension. Then $\mathcal{M}$ is a sub- $E$ - $B$-pair of $M_{S}^{*} \otimes_{S} \tilde{M}$. Put $\mathbf{D}_{B}(\mathcal{M})=\left(\mathcal{W}_{e}, \mathcal{W}_{\mathrm{dR}}^{+}\right)$.

A lifting of 1 in $\mathcal{W}_{e}$ is $\sum_{j} f_{j, z}^{*} \otimes f_{j}$, and a lifting of 1 in $\mathcal{W}_{\mathrm{dR}}^{+}$is $\sum_{j} g_{j, z}^{*} \otimes g_{j}$. We have

$$
\begin{aligned}
(\sigma-1) \sum_{j} f_{j, z}^{*} \otimes f_{j} & =\sigma\left(f_{1, z}^{*}, \ldots, f_{n, z}^{*}\right) \otimes \sigma\left(\begin{array}{c}
f_{1} \\
f_{2} \\
\vdots \\
f_{n}
\end{array}\right)-\left(f_{1, z}^{*}, \ldots, f_{n, z}^{*}\right) \otimes\left(\begin{array}{c}
f_{1} \\
f_{2} \\
\vdots \\
f_{n}
\end{array}\right) \\
& =\left(f_{1, z}^{*}, \ldots, f_{n, z}^{*}\right)\left(A_{e, \sigma}^{t}\right)^{-1} \otimes A_{e, \sigma}^{t}\left(1+z U_{e, \sigma}^{t}\right) \\
& =\left(f_{1, z}^{*}, \ldots, f_{n, z}^{*}\right) \otimes U_{e, \sigma}^{t} z\left(\begin{array}{c}
f_{1} \\
f_{2} \\
\vdots \\
f_{n}
\end{array}\right) .
\end{aligned}
$$

Similarly,

$$
(\sigma-1) \sum_{j} g_{j, z}^{*} \otimes g_{j}=\left(g_{1, z}^{*}, \ldots, g_{n, z}^{*}\right) \otimes\left(U_{\mathrm{dR}, \sigma}^{+}\right)^{t} z\left(\begin{array}{c}
g_{1} \\
g_{2} \\
\vdots \\
g_{n}
\end{array}\right),
$$

and

$$
\sum_{j} f_{j, z}^{*} \otimes f_{j}-\sum_{j} g_{j, z}^{*} \otimes g_{j}=\left(g_{1, z}^{*}, \ldots, g_{n, z}^{*}\right) \otimes U_{\mathrm{dR}}^{t} z\left(\begin{array}{c}
g_{1} \\
g_{2} \\
\vdots \\
g_{n}
\end{array}\right) .
$$

Hence the element in $H_{B}^{1}\left(\mathbf{D}_{B}\left(M^{*} \otimes_{E} M\right)\right)$ attached to the extension $\mathbf{D}_{B}(\mathcal{M})$ is $\left[c_{B}(\tilde{M})\right]$.

A similar computation shows that the element in $H_{\Phi \Gamma}^{1}\left(M^{*} \otimes_{E} M\right)$ attached to the extension $\mathcal{M}$ is $\left[c_{\Phi \Gamma}(\tilde{M})\right]$. Now (C) follows. 


\section{The Reciprocity Law and an Application}

\subsection{Reciprocity law}

In [14, Section 2] using local class field theory Zhang precisely described the perfect pairing

$$
H^{1}\left(G_{K}, E\right) \times H^{1}\left(G_{K}, E(1)\right) \rightarrow H^{2}\left(G_{K}, E(1)\right) .
$$

We recall it below.

The Kummer theory gives us a canonical isomorphism so called the Kummer map

$$
\begin{aligned}
\lim _{\leftarrow}\left(K^{\times} /\left(K^{\times}\right)^{p^{n}}\right) \otimes \mathbf{z}_{p} E & \rightarrow H^{1}\left(G_{K}, E(1)\right) \\
\sum_{i} \alpha_{i} \otimes a_{i} & \mapsto \sum_{i} a_{i}\left[\left(\alpha_{i}\right)\right] .
\end{aligned}
$$

Here $(\alpha)$ is the 1-cocycle such that

$$
\frac{g(\sqrt[p]{\alpha})}{\alpha}=\varepsilon_{n}^{\left(\alpha_{g}\right)}
$$

for $\alpha \in K^{\times}$and $g \in G_{K}$, where $(\sqrt[n+1]{\alpha})^{p}=\sqrt[p]{\alpha}$. Combining the Kummer map and the exponent map

$$
\exp : p \mathfrak{o}_{K} \rightarrow K^{\times}
$$

and extending it by linearity we obtain an embedding from $K \otimes_{\mathbf{Q}_{p}} E$ to $H^{1}\left(G_{K}, E(1)\right)$, again denoted by exp. Then we have

$$
H^{1}\left(G_{K}, E(1)\right)=\exp \left(K \otimes \mathbf{Q}_{p} E\right) \oplus E \cdot[(p)]
$$

Let $\operatorname{Hom}\left(G_{K}, E\right)$ be the group of additive characters of $G_{K}$ with values in $E$. As the action of $G_{K}$ on $E$ is trivial, $H^{1}\left(G_{K}, E\right)$ is naturally isomorphic to $\operatorname{Hom}\left(G_{K}, E\right)$. Let $\psi_{0}: G_{K} \rightarrow E$ be the additive character that vanishes on the inertial subgroup of $G_{K}$ and maps the geometrical Frobenius to $\left[K_{0}: \mathbf{Q}_{p}\right]$. For any $\tau \in \operatorname{Emb}(K, E)$ let $\psi_{\tau}$ be the composition $\left.\tau \circ \log \circ \operatorname{rec}_{K}^{-12}\right]$, where log

\footnotetext{
${ }^{2}$ Since the character $\psi_{\tau}$ of the Weil group $W_{K}$ sends any lifting of the $q$ th power Frobenius to 0 , it can be extended to a character of $G_{K}$ which is again denoted by $\psi_{\tau}$
} 
is normalized such that $\log (p)=0$. Then $\left\{\psi_{0}, \psi_{\tau}: \tau \in \operatorname{Emb}(K, E)\right\}$ is an E-basis of $H^{1}\left(G_{K}, E\right)$.

Lemma 5.1 (Zhang, Proposition 2.1). The cup product of

$a_{0} \psi_{0}+\sum_{\tau \in \operatorname{Emb}(K, E)} a_{\tau} \psi_{\tau}\left(a_{0}, a_{\tau} \in E\right)$ and $b_{0}[(p)]+\exp (b)\left(b_{0} \in E, b \in\right.$ $\left.K \otimes_{\mathbf{Q}_{p}} E\right)$ is

$$
\left(a_{0} b_{0}-\operatorname{tr}_{K / \mathbf{Q}_{p}}\left(\left(a_{\tau}\right)_{\tau} \cdot b\right)\right)\left(\psi_{0} \cup[(p)]\right)
$$

Here, $\left(a_{\tau}\right)_{\tau}$ is considered as an element in $K \otimes_{\mathbf{Q}_{p}} E$ via the isomorphism (2.1).

Lemma 5.2. For $\lambda_{0}, \lambda_{\tau} \in E(\tau \in \operatorname{Emb}(K, E))$, the extension of $E$ (as a trivial $G_{K}$-module) by $E$ corresponding to the cocycle $\lambda_{0} \psi_{0}+\sum_{\tau \in \operatorname{Emb}(K, E)} \lambda_{\tau} \psi_{\tau}$ is de Rham if and only if $\lambda_{\tau}=0$ for each $\tau$.

Proof. By [11, Lemma 4.3], the subspace of extensions of $E$ by $E$ that are de Rham is 1-dimensional, and so consists of those corresponding to the cocycles $\lambda_{0} \psi_{0}\left(\lambda_{0} \in E\right)$.

\subsection{An auxiliary formula}

Let $\overrightarrow{\mathcal{L}}=\left(\mathcal{L}_{\sigma}\right)_{\sigma: K \hookrightarrow E}$ be a vector. We consider $\overrightarrow{\mathcal{L}}$ as an element of $K \otimes_{\mathbf{Q}_{p}} E$ via the isomorphism (2.1).

Let $D$ be a filtered $E$ - $(\varphi, N)$-module: the underlying $E$ - $(\varphi, N)$-module $D$ is a $\left(K_{0} \otimes_{\mathbf{Q}_{p}} E\right)$-module with a basis $\left\{f_{1}, f_{2}, f_{3}\right\}$ such that

$$
\varphi^{\left[K_{0}: \mathbf{Q}_{p}\right]} f_{1}=p^{-\left[K_{0}: \mathbf{Q}_{p}\right]} f_{1}, \varphi^{\left[K_{0}: \mathbf{Q}_{p}\right]} f_{2}=f_{2}, \varphi^{\left[K_{0}: \mathbf{Q}_{p}\right]} f_{3}=f_{3},
$$

and

$$
N\left(f_{1}\right)=0, N\left(f_{2}\right)=-f_{1}, N\left(f_{3}\right)=f_{1} ;
$$

the filtration on

$$
K \otimes_{K_{0}} D=\left(K \otimes_{\mathbf{Q}_{p}} E\right) f_{1} \oplus\left(K \otimes_{\mathbf{Q}_{p}} E\right) f_{2} \oplus\left(K \otimes_{\mathbf{Q}_{p}} E\right) f_{3}
$$

satisfies

$$
\mathrm{Fil}^{i} D=\left\{\begin{array}{cc}
\left(K \otimes_{\mathbf{Q}_{p}} E\right)\left(f_{2}-\overrightarrow{\mathcal{L}} f_{1}\right) \oplus\left(K \otimes_{\mathbf{Q}_{p}} E\right)\left(f_{3}+\overrightarrow{\mathcal{L}} f_{1}\right) & \text { if } i=0 \\
0 & \text { if } i>0
\end{array}\right.
$$


Let $\pi_{i}$ be the projection map

$$
\mathbf{X}_{\log }(D) \rightarrow \mathbf{B}_{\log , E}, \quad \sum_{j=1}^{3} a_{j} f_{j} \mapsto a_{i} .
$$

Lemma 5.3. Let $c: G_{K} \rightarrow \mathbf{X}_{\log }(D)$ be a 1-cocycle whose class in $H^{1}\left(G_{K}\right.$, $\left.\mathbf{X}_{\log }(D)\right)$ belongs to $\operatorname{ker}\left(H^{1}\left(G_{K}, \mathbf{X}_{\log }(D)\right) \rightarrow H^{1}\left(G_{K}, \mathbf{X}_{\mathrm{dR}}(D)\right)\right)$. Then there exist

$$
\gamma_{2,0}, \gamma_{2, \tau}, \gamma_{3,0}, \gamma_{3, \tau} \in E
$$

$(\tau \in \operatorname{Emb}(K, E))$ such that

$$
\pi_{2}(c)=\gamma_{2,0} \psi_{0}+\sum_{\tau \in \operatorname{Emb}(K, E)} \gamma_{2, \tau} \psi_{\tau}
$$

and

$$
\pi_{3}(c)=\gamma_{3,0} \psi_{0}+\sum_{\tau \in \operatorname{Emb}(K, E)} \gamma_{3, \tau} \psi_{\tau}
$$

Furthermore,

$$
\gamma_{2,0}-\gamma_{3,0}=\sum_{\tau \in \operatorname{Emb}(K, E)} \mathcal{L}_{\tau}\left(\gamma_{2, \tau}-\gamma_{3, \tau}\right)
$$

In our proof of Lemma 5.3 we need the following

Lemma 5.4. Let $D$ be an $E$ - $(\varphi, N)$-module. If $\mathrm{Fil}_{1}$ and $\mathrm{Fil}_{2}$ are two filtrations on $K \otimes_{K_{0}} D$ such that $\mathrm{Fil}_{1}^{0}\left(K \otimes_{K_{0}} D\right)=\mathrm{Fil}_{2}^{0}\left(K \otimes_{K_{0}} D\right)$, then the kernel of

$$
H^{1}\left(G_{K}, \mathbf{X}_{\log }(D)\right) \rightarrow H^{1}\left(G_{K}, \mathbf{X}_{\mathrm{dR}}\left(D, \mathrm{Fil}_{1}\right)\right)
$$

coincides with the kernel of

$$
H^{1}\left(G_{K}, \mathbf{X}_{\log }(D)\right) \rightarrow H^{1}\left(G_{K}, \mathbf{X}_{\mathrm{dR}}\left(D, \mathrm{Fil}_{2}\right)\right)
$$

Proof. The proof is similar to that of [13, Proposition 2.5]

Proof of Lemma 5.3. The argument is similar to the proof of [13, Lemma 5.1]. We only give a sketch.

Write $c_{\sigma}=\lambda_{1, \sigma} f_{1}+\lambda_{2, \sigma} f_{2}+\lambda_{3, \sigma} f_{3}$. As $c$ takes values in $\mathbf{X}_{\log }(D)$, we have $\lambda_{2, \sigma}, \lambda_{3, \sigma} \in E$. This ensures the existence of $\gamma_{2,0}, \gamma_{2, \tau}, \gamma_{3,0}, \gamma_{3, \tau}$. 
Let $F i l$ be the filtration on $D$ such that $F i l^{-1} D=D$ and $F i l^{i} D=$ $\mathrm{Fil}^{i} D$ if $i \geq 0$. Then $(D, F i l)$ is admissible. Let $V$ be the semistable $E$ representation of $G_{K}$ attached to $D_{V}=(D, F i l)$. By Lemma [5.4, [c] is in the kernel of $H^{1}\left(G_{K}, \mathbf{X}_{\log }\left(D_{V}\right)\right) \rightarrow H^{1}\left(G_{K}, \mathbf{X}_{\mathrm{dR}}\left(D_{V}\right)\right)$ and so there exists a 1-cocycle $c^{(1)}: G_{K} \rightarrow V$ such that the image of $\left[c^{(1)}\right]$ by $H^{1}\left(G_{K}, V\right) \rightarrow$ $H^{1}\left(G_{K}, \mathbf{X}_{\log }\left(D_{V}\right)\right)$ is $[c]$.

We form the following commutative diagram

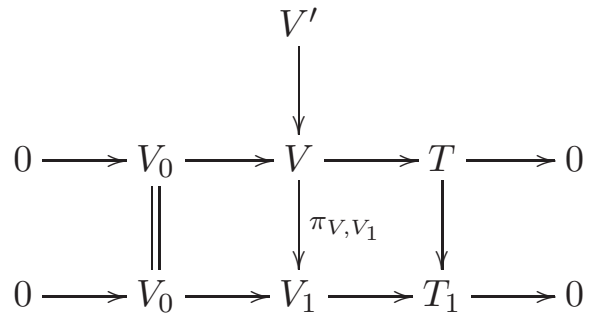

with the horizontal lines being exact, where $V_{0}$ (resp. $V^{\prime}$ ) is the subrepresentation of $V$ corresponding to the filtered $E-(\varphi, N)$-submodule of $D_{V}$ generated by $f_{1}$ (resp. by $f_{2}+f_{3}$ ) which is admissible. From (5.1) we obtain the following commutative diagram

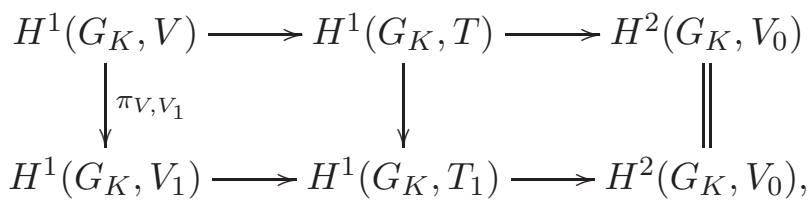

where the horizontal lines are exact.

Write $c^{(2)}$ for the 1-cocycle $G_{K} \stackrel{c^{(1)}}{\longrightarrow} V \rightarrow T \rightarrow T_{1}$. By a simple computation we obtain

$$
\left[c^{(2)}\right]=\left[\left(\left(\gamma_{2,0}-\gamma_{3,0}\right) \psi_{0}+\sum_{\tau \in \operatorname{Emb}(K, E)}\left(\gamma_{2, \tau}-\gamma_{3, \tau}\right) \psi_{\tau}\right) \bar{f}_{2}\right]
$$

where $\bar{f}_{2}$ is the image of $f_{2} \in V$ in $T_{1}$. Note that $T_{1}$ is isomorphic to $E$, and $V_{0}$ is isomorphic to $E(1)$. Being the image of $\left[\pi_{V, V_{1}}\left(c^{(1)}\right)\right]$ in $H^{1}\left(T_{1}\right),\left[c^{(2)}\right]$ lies in the kernel of $H^{1}\left(G_{K}, T_{1}\right) \rightarrow H^{2}\left(G_{K}, V_{0}\right)$. By [14, Lemma 5.5], as an extension of $E$ by $E(1), V_{1}$ corresponds to the element $[(p)]+\exp (\overrightarrow{\mathcal{L}})$. Now Lemma 5.1 yields our second assertion. 


\section{6. $L$-invariants}

Let $D$ be a filtered $E$ - $(\varphi, N)$-module of rank $n$. Fix a refinement $\mathcal{F}$ of $D$. Then $\mathcal{F}$ fixes an ordering $\alpha_{1}, \ldots, \alpha_{n}$ of the eigenvalues of $\varphi^{\left[K_{0}: \mathbf{Q}_{p}\right]}$ and an ordering $\vec{k}_{1}, \ldots, \vec{k}_{n}$ of the Hodge-Tate weights.

\subsection{The operator $N_{\mathcal{F}}$}

The operator $\varphi$ induces a $K_{0} \otimes_{\mathbf{Q}_{p}} E$-semilinear operator $\varphi_{\mathcal{F}}$ on $\operatorname{gr}_{\bullet}^{\mathcal{F}} D=$ $\bigoplus_{i=1}^{n} \mathcal{F}_{i} D / \mathcal{F}_{i-1} D$

We define a $K_{0} \otimes_{\mathbf{Q}_{p}} E$-linear operator $N_{\mathcal{F}}$ on $\operatorname{gr}_{\bullet}^{\mathcal{F}} D$. The definition is similar to the one defined in [13], so we omit some details.

For any $i \in\{1, \ldots, n\}$, if $N\left(\mathcal{F}_{i} D\right)=N\left(\mathcal{F}_{i-1} D\right)$, we demand that $N_{\mathcal{F}}$ $\operatorname{maps} \operatorname{gr}_{i}^{\mathcal{F}} D$ to zero.

Now we assume that $N\left(\mathcal{F}_{i} D\right) \supsetneq N\left(\mathcal{F}_{i-1} D\right)$. Let $j$ be the minimal integer such that

$$
N\left(\mathcal{F}_{i} D\right) \subseteq N\left(\mathcal{F}_{i-1} D\right)+\mathcal{F}_{j} D
$$

Proposition 6.1. $N\left(\mathcal{F}_{i-1} D\right) \cap \mathcal{F}_{j} D=N\left(\mathcal{F}_{i-1} D\right) \cap \mathcal{F}_{j-1} D$.

Proof. Note that $\mathcal{F}_{j} D, \mathcal{F}_{j-1} D, N\left(\mathcal{F}_{i-1} D\right)+\mathcal{F}_{j} D$ and $N\left(\mathcal{F}_{i-1} D\right)+\mathcal{F}_{j-1} D$ are stable by $\varphi$. Thus $\left(N\left(\mathcal{F}_{i-1} D\right)+\mathcal{F}_{j} D\right) /\left(N\left(\mathcal{F}_{i-1} D\right)+\mathcal{F}_{j-1} D\right)$ is a $\varphi$ module, and so must be free over $K_{0} \otimes_{\mathbf{Q}_{p}} E$. Hence the map

$$
\mathcal{F}_{j} D / \mathcal{F}_{j-1} D \rightarrow\left(N\left(\mathcal{F}_{i-1} D\right)+\mathcal{F}_{j} D\right) /\left(N\left(\mathcal{F}_{i-1} D\right)+\mathcal{F}_{j-1} D\right)
$$

is an isomorphism. It follows that $N\left(\mathcal{F}_{i-1} D\right) \cap \mathcal{F}_{j} D=N\left(\mathcal{F}_{i-1} D\right) \cap \mathcal{F}_{j-1} D$.

The operator $N$ induces a $K_{0} \otimes_{\mathbf{Q}_{p}} E$-linear map

$$
\mathcal{F}_{i} D / \mathcal{F}_{i-1} D \rightarrow\left(N\left(\mathcal{F}_{i-1} D\right)+\mathcal{F}_{j} D\right) /\left(N\left(\mathcal{F}_{i-1} D\right)+\mathcal{F}_{j-1} D\right)
$$

We define the map $N_{\mathcal{F}}: \operatorname{gr}_{i}^{\mathcal{F}} D \rightarrow \operatorname{gr}_{j}^{\mathcal{F}} D$ to be the composition of this map and the inverse of (6.1).

Finally we extend $N_{\mathcal{F}}$ to the whole $\operatorname{gr}_{\bullet}^{\mathcal{F}} D$ by $K_{0} \otimes_{\mathbf{Q}_{p}} E$-linearity. Note that $N_{\mathcal{F}} \varphi_{\mathcal{F}}=p \varphi_{\mathcal{F}} N_{\mathcal{F}}$. By definition, for any $i$ we have either $N\left(\operatorname{gr}_{i}^{\mathcal{F}} D\right)=0$ or $N\left(\operatorname{gr}_{i}^{\mathcal{F}} D\right)=\operatorname{gr}_{j}^{\mathcal{F}} D$ for some $j$. 
Definition 6.2. For $j \in\{1, \ldots, n-1\}$ we say that $j$ is marked (or a marked index) for $\mathcal{F}$ if there is some $i \in\{2, \ldots, n\}$ such that $N_{\mathcal{F}}\left(\operatorname{gr}_{i}^{\mathcal{F}} D\right)=\operatorname{gr}_{j}^{\mathcal{F}} D$.

Note that $i$ and $j$ in the above definition are determined by each other. We write $i=t_{\mathcal{F}}(j)$ and $j=s_{\mathcal{F}}(i)$.

Proposition 6.3. The following two assertions are equivalent:

(a) $s$ is marked and $t=t_{\mathcal{F}}(s)$.

(b) $N \mathcal{F}_{t-1} D \cap \mathcal{F}_{s} D=N \mathcal{F}_{t-1} D \cap \mathcal{F}_{s-1} D$ and $N \mathcal{F}_{t} D \cap \mathcal{F}_{s} D \supsetneq N \mathcal{F}_{t} D$ $\cap \mathcal{F}_{s-1} D$.

Proof. We have already seen that, if (国) holds, then (b) holds. Conversely, we assume that (b) holds. Then $N \mathcal{F}_{t} D \cap \mathcal{F}_{s} D \supsetneq N \mathcal{F}_{t-1} D \cap \mathcal{F}_{s} D$. Thus $N \mathcal{F}_{t} D \supsetneq N \mathcal{F}_{t-1} D$.

We show that $N \mathcal{F}_{t} D \varsubsetneqq N \mathcal{F}_{t-1} D+\mathcal{F}_{s-1} D$. If it is not true, then there exists $y \in \mathcal{F}_{t} D \backslash \mathcal{F}_{t-1} D$ which is a lifting of a basis of $\operatorname{gr}_{t}^{\mathcal{F}} D$ over $K_{0} \otimes_{\mathbf{Q}_{p}} E$ such that $N(y) \in \mathcal{F}_{s-1} D$. For any $z \in \mathcal{F}_{t} D$, write $z=w+\lambda y$ with $w \in$ $\mathcal{F}_{t-1} D$ and $\lambda \in K_{0} \otimes_{\mathbf{Q}_{p}} E$. If $N(z)$ is in $\mathcal{F}_{s} D$, then $N(w)$ is also in $\mathcal{F}_{s} D$. But $N \mathcal{F}_{t-1} D \cap \mathcal{F}_{s} D=N \mathcal{F}_{t-1} D \cap \mathcal{F}_{s-1} D$. Thus $N(w)$ is in $\mathcal{F}_{s-1} D$, which implies that $N(z)=N(w)+\lambda N(y)$ is also in $\mathcal{F}_{s-1} D$. So, $N \mathcal{F}_{t} D \cap \mathcal{F}_{s} D=$ $N \mathcal{F}_{t} D \cap \mathcal{F}_{s-1} D$, a contradiction.

From $N \mathcal{F}_{t} D \cap \mathcal{F}_{s} D \supsetneq N \mathcal{F}_{t-1} D \cap \mathcal{F}_{s} D$ we see that there is $x \in \mathcal{F}_{t} D \backslash \mathcal{F}_{t-1} D$ such that $N(x) \in \mathcal{F}_{s} D$. We must have $N \mathcal{F}_{t} D \subseteq N \mathcal{F}_{t-1} D+\mathcal{F}_{s} D$. Otherwise, let $j$ be the smallest integer such that $N \mathcal{F}_{t} D \subseteq N \mathcal{F}_{t-1} D+\mathcal{F}_{j} D$ and assume that $j>s$. Then $N_{\mathcal{F}}\left(x+\mathcal{F}_{t-1} D\right)=0$, which contradicts the fact that $N_{\mathcal{F}}: \operatorname{gr}_{t}^{\mathcal{F}} D \rightarrow \operatorname{gr}_{j}^{\mathcal{F}} D$ is an isomorphism.

\subsection{Strongly marked indices and $\mathcal{L}$-invariants}

Assume that $s$ is marked for $\mathcal{F}$ and $t=t_{\mathcal{F}}(s)$. We consider the decompositions

$$
\mathcal{F}_{t} D / \mathcal{F}_{s-1} D=\left(K_{0} \otimes_{\mathbf{Q}_{p}} E\right) \cdot \bar{e}_{s} \oplus L \oplus\left(K_{0} \otimes_{\mathbf{Q}_{p}} E\right) \bar{e}_{t}
$$

that satisfy the following conditions:

- $\overline{\mathcal{F}}_{1}\left(\mathcal{F}_{t} D / \mathcal{F}_{s-1} D\right)=\left(K_{0} \otimes_{\mathbf{Q}_{p}} E\right) \bar{e}_{s}$ and $\overline{\mathcal{F}}_{t-s}\left(\mathcal{F}_{t} D / \mathcal{F}_{s-1} D\right)=\left(K_{0} \otimes_{\mathbf{Q}_{p}}\right.$ $E) \bar{e}_{s} \oplus L$, where $\overline{\mathcal{F}}$ is the refinement on $\mathcal{F}_{t} D / \mathcal{F}_{s-1} D$ induced by $\mathcal{F}$. 
- Both $L$ and $\left(K_{0} \otimes_{\mathbf{Q}_{p}} E\right) \bar{e}_{s} \oplus\left(K_{0} \otimes \mathbf{Q}_{p} E\right) \bar{e}_{t}$ are stable by $\varphi$ and $N$; $\varphi^{\left[K_{0}: \mathbf{Q}_{p}\right]}\left(\bar{e}_{t}\right)=\alpha_{t} \bar{e}_{t}$ and $N\left(\bar{e}_{t}\right)=\bar{e}_{s}$.

Such a decomposition is called an s-decomposition.

Remark 6.4. $s$-decompositions may be not exist. However, if $\varphi$ is semisimple, then $s$-decompositions always exist (see [13]).

Let dec denote an $s$-decomposition $\mathcal{F}_{t} D / \mathcal{F}_{s-1} D=E \bar{e}_{s} \oplus L \oplus E \bar{e}_{t}$.

There is a natural isomorphism $E \bar{e}_{s} \oplus E \bar{e}_{t} \rightarrow\left(\mathcal{F}_{t} D / \mathcal{F}_{s-1} D\right) / L$ of $(\varphi, N)$ modules. Usually the filtration on the filtered $E$ - $(\varphi, N)$-submodule $E \bar{e}_{s} \oplus E \bar{e}_{t}$ and that on $\left(\mathcal{F}_{t} D / \mathcal{F}_{s-1} D\right) / L$ are different.

When these two filtrations satisfy certain compatible condition, we say the decomposition dec is perfect. Precisely, we say that dec is perfect if for any $\tau: K \hookrightarrow E$ we have $k_{s, \tau}<k_{t, \tau}$, and if there exist $k_{s, \tau}^{\prime}, k_{t, \tau}^{\prime}$ and $\mathcal{L}_{\text {dec }, \tau} \in E$ satisfying $k_{s, \tau} \leq k_{s, \tau}^{\prime}<k_{t, \tau}^{\prime} \leq k_{t, \tau}$ such that the following conditions hold.

- The filtration on the filtered $E$ - $(\varphi, N)$-submodule $E \bar{e}_{s} \oplus E \bar{e}_{t}$ satisfies

$$
\mathrm{Fil}_{\tau}^{i}\left(E \bar{e}_{s} \oplus E \bar{e}_{t}\right)= \begin{cases}E \bar{e}_{s, \tau} \oplus E \bar{e}_{t, \tau} & \text { if } i \leq k_{s, \tau}, \\ E\left(\bar{e}_{t, \tau}+\mathcal{L}_{\mathrm{dec}, \tau} \bar{e}_{s, \tau}\right) & \text { if } k_{s, \tau}<i \leq k_{t, \tau}^{\prime}, \\ 0 & \text { if } i>k_{t, \tau}^{\prime},\end{cases}
$$

- The filtration on the quotient of $\mathcal{F}_{t} D / \mathcal{F}_{s-1} D$ by $L$ satisfies

$$
\mathrm{Fil}_{\tau}^{i} \mathcal{F}_{t} D / \mathcal{F}_{s-1} D= \begin{cases}E \bar{e}_{s, \tau} \oplus E \bar{e}_{t, \tau} & \text { if } i \leq k_{s, \tau}^{\prime} \\ E\left(\bar{e}_{t}+\mathcal{L}_{\operatorname{dec}, \tau} \bar{e}_{s}\right) & \text { if } k_{s, \tau}^{\prime}<i \leq k_{t, \tau} \\ 0 & \text { if } i>k_{t, \tau}\end{cases}
$$

where the images of $\bar{e}_{s}$ and $\bar{e}_{t}$ in $\mathcal{F}_{t} D / \mathcal{F}_{s-1} D$ are again denoted by $\bar{e}_{s}$ and $\bar{e}_{t}$.

Definition 6.5. If there exists a perfect $s$-decomposition, we say that $s$ is strongly marked (or a strongly marked index). In this case we attached to each pair $(s, t)$ with $t=t_{\mathcal{F}}(s)$ an invariant $\overrightarrow{\mathcal{L}}_{\mathcal{F}, s, t}=\left(\mathcal{L}_{\text {dec }, \tau}\right)_{\tau}$, where dec is a perfect $s$-decomposition. Proposition 6.6 below tells us that $\overrightarrow{\mathcal{L}}_{\mathcal{F}, s, t}$ is independent of the choice of perfect $s$-decompositions. We call $\overrightarrow{\mathcal{L}}_{\mathcal{F}, s, t}$ the Fontaine-Mazur $\mathcal{L}$-invariant associated to $(\mathcal{F}, s, t)$, and denote $\mathcal{L}_{\text {dec }, \tau}$ by $\mathcal{L}_{\mathcal{F}, s, t, \tau}$. 
In the case of $t=s+1, s$ is strongly marked if and only if $k_{s, \tau}<k_{t, \tau}$ for all $\tau$.

Proposition 6.6. If $\operatorname{dec}_{1}$ and $\operatorname{dec}_{2}$ are two perfect $s$-decompositions, then $\mathcal{L}_{\operatorname{dec}_{1}, \tau}=\mathcal{L}_{\operatorname{dec}_{2}, \tau}$ for any $\tau$.

Proof. The argument is similar to the proof of [13, Proposition 4.9].

Let $D^{*}$ be the filtered $E$ - $(\varphi, N)$-module that is the dual of $D$. Let $\breve{\mathcal{F}}$ be the refinement on $D^{*}$ such that

$$
\check{\mathcal{F}}_{i} D^{*}:=\left(\mathcal{F}_{n-i} D\right)^{\perp}=\left\{y \in D^{*}:\langle y, x\rangle=0 \text { for all } x \in \mathcal{F}_{n-i} D\right\} .
$$

We call $\check{\mathcal{F}}$ the dual refinement of $\mathcal{F}$.

If $L \subset M$ are submodules of $D$, then $M^{\perp} \subset L^{\perp}$. The pairing $\langle\cdot, \cdot\rangle$ : $L^{\perp} \times M$ induces a non-degenerate pairing on $L^{\perp} / M^{\perp} \times M / L$, so that we can identify $L^{\perp} / M^{\perp}$ with the dual of $M / L$ naturally. In particular, $\operatorname{gr}_{i}^{\breve{\mathcal{F}}} D^{*}$ is naturally isomorphic to the dual of $\operatorname{gr}_{n+1-i}^{\mathcal{F}} D$. Thus $\operatorname{gr}_{\bullet}^{\check{\mathcal{F}}} D^{*}$ is naturally isomorphic to the dual of $\mathrm{gr}_{\bullet}^{\mathcal{F}} D$.

\section{Proposition 6.7.}

(a) $N_{\breve{\mathcal{F}}}$ is dual to $-N_{\mathcal{F}}$.

(b) $s$ is marked for $\mathcal{F}$ if and only if $n+1-t_{\mathcal{F}}(s)$ is marked for $\check{\mathcal{F}}$.

(c) $s$ is strongly marked for $\mathcal{F}$ if and only if $n+1-t_{\mathcal{F}}(s)$ is strongly marked for $\check{\mathcal{F}}$.

Proof. The proof of (国) is similar to that of [13, Proposition 4.14]. The proof of (B) is similar to that of [13, Proposition 4.13]. The proof of ([C) is similar to that of 13, Proposition 4.15 (a)].

\section{Projection Vanishing Property}

Put $S=E[Z] /\left(Z^{2}\right)$. Let $z$ be the closed point defined by the maximal ideal $(Z)$ of $S$.

Let $W=\left(W_{e}, W_{\mathrm{dR}}^{+}\right)$be an $S$ - $B$-pair. Let $\left\{w_{1}, \ldots, w_{n}\right\}$ be a $\mathbf{B}_{e, S}$-basis of $W_{e}$. Suppose that $W$ admits a triangulation Fil. . Let $\left(\delta_{1}, \ldots, \delta_{n}\right)$ be the corresponding triangulation parameters. Then for each $i=1, \ldots, n$ there 
exists a continuous additive character $\epsilon_{i}$ of $K^{\times}$with values in $E$ such that $\delta_{i}=\delta_{i, z}\left(1+Z \epsilon_{i}\right)$.

Suppose that $W_{z}$, the evaluation of $W$ at $z$, is semistable, and let $D_{z}$ be the filtered $E-(\varphi, N)$-module attached to $W_{z}$. Let $\mathcal{F}$ be the refinement of $D_{z}$ corresponding to the induced triangulation of $W_{z}$, and let $\left\{e_{1, z}, e_{2, z}, \ldots, e_{n, z}\right\}$ be a $\left(K_{0} \otimes_{\mathbf{Q}_{p}} E\right)$-basis of $D_{z}$ that is compatible with $\mathcal{F}$ i.e. $\mathcal{F}_{i} D=\left(K_{0} \otimes_{\mathbf{Q}_{p}}\right.$ $E) e_{1, z} \oplus \cdots \oplus\left(K_{0} \otimes_{\mathbf{Q}_{p}} E\right) e_{i, z}$. Let $\alpha_{i, z} \in E$ be such that $\varphi^{\left[K_{0}: \mathbf{Q}_{p}\right]}\left(e_{i, z}\right)=$ $\alpha_{i, z} e_{i, z} \bmod \mathcal{F}_{i-1}$.

Let $x_{i j} \in \mathbf{B}_{\log , E}(i, j=1, \ldots, n)$ be such that

$$
e_{i, z}=x_{1 i} w_{1, z}+\cdots+x_{n i} w_{n, z}
$$

Then $X=\left(x_{i j}\right)$ is in $\mathrm{GL}_{n}\left(\mathbf{B}_{\log , E}\right)$. Write the matrix of $\sigma \in G_{K}$ with respect to the basis $\left\{w_{1}, \ldots, w_{n}\right\}$ by $\left(I_{n}+Z U_{e, \sigma}\right) A_{e, \sigma}$. As $e_{1, z}, \ldots, e_{n, z}$ are fixed by $G_{K}$, we have $X^{-1} A_{e, \sigma} \sigma(X)=I_{n}$ for all $\sigma \in G_{K}$.

For $i=1, \ldots, n$ put $e_{i}=x_{1 i} w_{1}+\cdots+x_{n i} w_{n}$. Then $\left\{e_{1}, \ldots, e_{n}\right\}$ is a basis of $\mathbf{B}_{\log , S} \otimes_{S} W_{e}$ over $\mathbf{B}_{\log , S}$.

Lemma 7.1. If $T$ is the matrix of $\varphi_{D_{z}}$ for the basis $\left\{e_{1, z}, \ldots, e_{n, z}\right\}$, then $T$ is also the matrix of $\varphi_{\mathbf{B}_{\log , S} \otimes_{S} W_{e}}$ for the basis $\left\{e_{1}, \ldots, e_{n}\right\}$.

Proof. The assertion follows from the definition of $\left\{e_{1}, \ldots, e_{n}\right\}$ and the fact that $w_{1, z}, \ldots, w_{n, z}, w_{1}, \ldots, w_{n}$ are fixed by $\varphi$.

In Section 4.1 we attach to $W$ an element $c_{B}(W)$ in $H_{B}^{1}\left(W_{z}^{*} \otimes W_{z}\right)$. Consider the composition

$H_{B}^{1}\left(W_{z}^{*} \otimes W_{z}\right) \rightarrow H^{1}\left(G_{K}, W_{e, z}^{*} \otimes_{\mathbf{B}_{e, E}} W_{e, z}\right) \rightarrow H^{1}\left(G_{K}, \mathbf{B}_{\log , E} \otimes_{E}\left(D_{z}^{*} \otimes D_{z}\right)\right)$.

As the matrix of $\sigma \in G_{K}$ for the basis $\left\{e_{1}, \ldots, e_{n}\right\}$ is $I_{n}+Z X^{-1} U_{e, \sigma} X$, from the discussion in Section 4 we see that the image of $c_{B}$ in $H^{1}\left(G_{K}, \mathbf{B}_{\log , E} \otimes_{E}\right.$ $\left.\left(D_{z}^{*} \otimes D_{z}\right)\right)$ is the class of the 1-cocycle

$$
\left(U_{e, \sigma}\right)_{i j} w_{j, z}^{*} \otimes w_{i, z}=\left(X^{-1} U_{e, \sigma} X\right)_{i j} e_{j, z}^{*} \otimes e_{i, z}
$$

Let $\pi_{h \ell}$ be the projection

$$
\mathbf{B}_{\log , E} \otimes_{E}\left(D_{z}^{*} \otimes D_{z}\right) \rightarrow \mathbf{B}_{\log , E}, \quad \sum_{j, i} b_{j i} e_{j, z}^{*} \otimes e_{i, z} \mapsto b_{h \ell}
$$


For $h=1, \ldots, n$, let $\epsilon_{h}^{\prime}$ be the additive character of $G_{K}$ such that $\epsilon_{h}^{\prime}$ 。 $\operatorname{rec}_{K}(p)=0$ and $\left.\epsilon_{h}^{\prime} \circ \operatorname{rec}_{K}\right|_{\mathfrak{o}_{K}^{\times}}=\left.\epsilon_{h}\right|_{\mathfrak{o}_{K}^{\times}}$.

\section{Theorem 7.2.}

(a) For any pair of integers $(h, \ell)$ such that $h<\ell$ we have $\pi_{h \ell}([c])=0$.

(b) For any $h=1, \ldots, n, \pi_{h, h}([c])$ coincides with the image of $\left[\epsilon_{h}^{\prime}\right]$ in $H^{1}\left(G_{K}, \mathbf{B}_{\log , E}\right)$.

We call (a) the projection vanishing property.

Proof. The filtered $E-(\varphi, N)$-module attached to $W_{z} /$ Fil $_{h-1} W_{z}$ is $D_{z} / \mathcal{F}_{h-1} D_{z}$. We denote the image of $e_{\ell, z}(\ell \geq h)$ in $D_{z} / \mathcal{F}_{h-1} D_{z}$ again by $e_{\ell, z}$.

Let $\delta_{h}^{\prime}$ be the character of $G_{K}$ such that $\delta_{h}^{\prime}=1+Z \epsilon_{h}^{\prime}$. By Lemma 3.5 there exists an element

$$
x \in\left(\mathbf{B}_{\max , E} \otimes \mathbf{B}_{e, E}\left(W / \mathrm{Fil}_{h-1} W\right)_{e}\right)^{G_{K}=\delta_{h}^{\prime}, \varphi^{\left[K_{0}: \mathbf{Q}_{p}\right]}=\alpha_{i, z}\left(1+Z v_{p}\left(\pi_{K}\right) \epsilon_{h}(p)\right)}
$$

whose image in $D_{z} / \mathcal{F}_{h-1} D_{z}$ is $e_{h, z}$. Write $x=e_{h}+Z \sum_{\ell \geq h} \lambda_{\ell} e_{\ell}$ with $\lambda_{\ell} \in$ $\mathbf{B}_{\log , E}$.

As the matrix of $\sigma \in G_{K}$ for the basis $\left\{e_{1}, \ldots, e_{n}\right\}$ is $I_{n}+Z X^{-1} U_{e, \sigma} X$, we have

$$
\begin{aligned}
{\left[1+Z \epsilon_{h}^{\prime}(\sigma)\right] x } & =\left[1+Z \epsilon_{h}^{\prime}(\sigma)\right]\left(e_{h}+Z \sum_{\ell \geq h} \lambda_{\ell} e_{\ell}\right) \\
& =\sigma(x)=e_{h}+Z \sum_{\ell \geq h}\left(X^{-1} U_{e, \sigma} X\right)_{\ell h} e_{\ell}+Z \sum_{\ell \geq h} \sigma\left(\lambda_{\ell}\right) e_{\ell} .
\end{aligned}
$$

For $\ell>h$, comparing the coefficients of $e_{\ell}$ we obtain

$$
\left(X^{-1} U_{e, \sigma} X\right)_{\ell h}=(1-\sigma) \lambda_{\ell},
$$

which shows (国). Similarly, comparing coefficients of $e_{h}$ we obtain

$$
\left(X^{-1} U_{e, \sigma} X\right)_{h h}-\epsilon_{h}^{\prime}(\sigma)=(1-\sigma) \lambda_{h},
$$

which implies (b). 


\section{The proof of Theorem 1.2}

We will need the following lemmas.

Lemma 8.1. The inclusion $E \hookrightarrow \mathbf{B}_{e, E}$ induces an isomorphism

$$
H^{1}\left(G_{K}, E\right) \stackrel{\sim}{\rightarrow} \operatorname{ker}\left(N: H^{1}\left(G_{K}, \mathbf{B}_{e, E}\right) \rightarrow H^{1}\left(G_{K}, \mathbf{B}_{\log , E}\right)\right)
$$

Proof. The proof is identical to that of [13, Corollary 1.4].

Lemma 8.2. The map $N: \mathbf{B}_{\log , E}^{\varphi=p} \rightarrow \mathbf{B}_{\log , E}^{\varphi=1}$ is surjective.

Proof. The proof is identical to that of [13, Lemma 1.2].

For the proof of Theorem 1.2 we may assume that $S=E[Z] /\left(Z^{2}\right)$, and $z$ is the closed point defined by the maximal ideal $(Z)$. Let $W$ be as in Theorem 1.2. Replacing $W$ by the $E$ - $B$-pair $\mathcal{F}_{t} W / \mathcal{F}_{s-1} W$ and replacing $\mathcal{F}$ by the induced refinement on $\mathcal{F}_{t} W / \mathcal{F}_{s-1} W$, we may assume that $s=1$ and $t=n=\operatorname{rank}_{\mathbf{B}_{e, E}}\left(W_{e}\right)$. Let $e_{1, z}, e_{2, z}, \ldots, e_{n, z}$ be a $K_{0} \otimes_{\mathbf{Q}_{p}} E$-basis of $D_{z}$ such that

$$
\left(K_{0} \otimes_{\mathbf{Q}_{p}} E\right) e_{1, z} \bigoplus L \bigoplus\left(K_{0} \otimes_{\mathbf{Q}_{p}} E\right) e_{n, z}
$$

with $L=\oplus_{i=2}^{n-1}\left(K_{0} \otimes_{\mathbf{Q}_{p}} E\right) e_{i, z}$ a perfect 1-decomposition of $D_{z}$ for $\mathcal{F}$ (see $\S 6.2$ for the meaning of perfect decompositions). Let $e_{1, z}^{*}, e_{2, z}^{*}, \ldots, e_{n, z}^{*}$ be the dual basis of $D_{z}^{*}$ over $K_{0} \otimes \mathbf{Q}_{p} E$.

Let $D_{1}$ be the quotient of $D_{z}$ by $L, D_{2}^{*}$ the quotient of $D_{z}^{*}$ by $\oplus_{i=2}^{n-1}\left(K_{0} \otimes \mathbf{Q}_{p}\right.$ $E) e_{i, z}^{*}$. Put $\mathscr{D}=D_{2}^{*} \otimes D_{1}$. The images of $e_{1, z}$ and $e_{n, z}$ in $D_{1}$ are again denoted by $e_{1, z}$ and $e_{n, z}$, and the images of $e_{1, z}^{*}$ and $e_{n, z}^{*}$ in $D_{2}^{*}$ are again denoted by $e_{1, z}^{*}$ and $e_{n, z}^{*}$ respectively. So $e_{1, z}^{*} \otimes e_{1, z}, e_{1, z}^{*} \otimes e_{n, z}, e_{n, z}^{*} \otimes e_{1, z}, e_{n, z}^{*} \otimes e_{n, z}$ form a $K_{0} \otimes \mathbf{Q}_{p} E$-basis of $\mathscr{D}$. Let $\mathscr{D}_{0}$ be the filtered $E$ - $(\varphi, N)$-submodule of $\mathscr{D}$ with a $K_{0} \otimes \mathbf{Q}_{p} E$-basis $\left\{e_{1, z}^{*} \otimes e_{1, z}, e_{n, z}^{*} \otimes e_{1, z}, e_{n, z}^{*} \otimes e_{n, z}\right\}$. Let $\mathscr{W}=\left(\mathscr{W}_{e}, \mathscr{W}_{\mathrm{dR}}^{+}\right)$ (resp. $\left.\mathscr{W}_{0}\right)$ be the $E$ - $B$-pair attached to $\mathscr{D}$ (resp. $\left.\mathscr{D}_{0}\right)$. Note that

$$
\begin{gathered}
\varphi^{\left[K_{0}: \mathbf{Q}_{p}\right]}\left(e_{1, z}^{*} \otimes e_{1, z}\right)=e_{1, z}^{*} \otimes e_{1, z}, \varphi^{\left[K_{0}: \mathbf{Q}_{p}\right]}\left(e_{n, z}^{*} \otimes e_{n, z}\right)=e_{n, z}^{*} \otimes e_{n, z}, \\
\varphi^{\left[K_{0}: \mathbf{Q}_{p}\right]}\left(e_{n, z}^{*} \otimes e_{1, z}\right)=p^{-\left[K_{0}: \mathbf{Q}_{p}\right]} e_{n, z}^{*} \otimes e_{1, z}
\end{gathered}
$$

and

$$
-N\left(e_{1, z}^{*} \otimes e_{1, z}\right)=N\left(e_{n, z}^{*} \otimes e_{n, z}\right)=e_{n, z}^{*} \otimes e_{1, z}, N\left(e_{n, z}^{*} \otimes e_{1, z}\right)=0
$$


Let $\overrightarrow{\mathcal{L}}_{\mathcal{F}}=\overrightarrow{\mathcal{L}}_{\mathcal{F}, s, t}$ be the $\mathcal{L}$-invariant defined in Definition 6.5, As (8.1) is a prefect decomposition, we have

$$
\begin{aligned}
\operatorname{Fil}^{0}\left(K \otimes_{K_{0}} \mathscr{D}\right)= & E e_{n, z}^{*} \otimes\left(e_{n, z}+\overrightarrow{\mathcal{L}}_{\mathcal{F}} e_{1, z}\right) \oplus E\left(e_{1, z}^{*}-\overrightarrow{\mathcal{L}}_{\mathcal{F}} e_{n, z}^{*}\right) \otimes e_{1, z} \\
& \oplus E\left(e_{1, z}^{*}-\overrightarrow{\mathcal{L}}_{\mathcal{F}} e_{n, z}^{*}\right) \otimes\left(e_{n, z}+\overrightarrow{\mathcal{L}}_{\mathcal{F}} e_{1, z}\right) .
\end{aligned}
$$

and

$$
\operatorname{Fil}^{0}\left(K \otimes_{K_{0}} \mathscr{D}_{0}\right)=E e_{n, z}^{*} \otimes\left(e_{n, z}+\overrightarrow{\mathcal{L}}_{\mathcal{F}} e_{1, z}\right) \oplus E\left(e_{1, z}^{*}-\overrightarrow{\mathcal{L}}_{\mathcal{F}} e_{n, z}^{*}\right) \otimes e_{1, z} .
$$

Consider $W$ as an infinitesimal deformation of $W_{z}$. In Section 4.2 we attach to this infinitesimal deformation an element $c_{B}(W)$ in $H_{B}^{1}\left(W_{z}^{*} \otimes W_{z}\right)$. Let $[c]$ be the image of $c_{B}(W)$ by the composition

$$
H_{B}^{1}\left(W_{z}^{*} \otimes W_{z}\right) \rightarrow H^{1}\left(G_{K}, W_{e, z}^{*} \otimes_{\mathbf{B}_{e, E}} W_{e, z}\right) \rightarrow H^{1}\left(G_{K}, \mathbf{B}_{\log , E} \otimes_{K_{0} \otimes \mathbf{Q}_{p} E}\left(D_{z}^{*} \otimes D_{z}\right)\right)
$$

and choose a 1-cocyle $c$ representing $[c]$. Write $c$ in the form

$$
c=\sum_{j, i} c_{j, i} e_{j, z}^{*} \otimes e_{i, z}
$$

with $c_{i, j}$ being a 1-cocycle of $G_{K}$ with values in $\mathbf{B}_{\log , E}$. By the projection vanishing property (Theorem $7.2(\underline{a})$ ) we have $\left[c_{1, n}\right]=0$.

Lemma 8.3. There exist $\xi_{1}, \xi_{n} \in \mathbf{B}_{e, E}$ and $\gamma_{1,0}, \gamma_{1, \tau}, \gamma_{n, 0}, \gamma_{n, \tau}(\tau \in \operatorname{Emb}(K, E))$ such that

$$
c_{1,1}(\sigma)=(\sigma-1) \xi_{1}+\gamma_{1,0} \psi_{0}(\sigma)+\sum_{\tau \in \operatorname{Emb}(K, E)} \gamma_{1, \tau} \psi_{\tau}(\sigma)
$$

and

$$
c_{n, n}(\sigma)=(\sigma-1) \xi_{n}+\gamma_{n, 0} \psi_{0}(\sigma)+\sum_{\tau \in \operatorname{Emb}(K, E)} \gamma_{n, \tau} \psi_{\tau}(\sigma)
$$

for any $\sigma \in G_{K}$.

Proof. Let $\bar{c}_{B}$ be the image of $c_{B}$ in $H_{B}^{1}(\mathscr{W})$, and let $\bar{c}$ be the 1-cocycle

$$
\bar{c}=\sum_{j, i \in\{1, n\}} c_{j, i} e_{j, z}^{*} \otimes e_{i, z}
$$


of $G_{K}$ with values in $\mathbf{B}_{\log , E} \otimes_{K_{0} \otimes \mathbf{Q}_{p} E} \mathscr{D}$. Then the image of $\bar{c}_{B}$ in

$$
H^{1}\left(G_{K}, \mathbf{B}_{\log , E} \otimes_{K_{0} \otimes_{\mathbf{Q}_{p}} E} \mathscr{D}\right)
$$

is $[\bar{c}]$.

Note that $\bar{c}$ has values in $\mathscr{W}_{e}=\left(\mathbf{B}_{\log , E} \otimes_{K_{0} \otimes \mathbf{Q}_{p} E} \mathscr{D}\right)^{\varphi=1, N=0}$. So, in particular $c_{1,1}$ and $c_{n, n}$ have values in $\mathbf{B}_{e, E}$. As $N \bar{c}=0$, we have

$$
N\left(c_{n, 1}\right)=c_{1,1}-c_{n, n}, \quad-N\left(c_{1,1}\right)=N\left(c_{n, n}\right)=c_{1, n}
$$

As $\left[c_{1, n}\right]=0$, the statement follows from Lemma 8.1.

Write $\delta_{i}=\delta_{i, z}\left(1+Z \epsilon_{i}\right)$. Let $\epsilon_{i}^{\prime}$ be the additive character of $G_{K}$ with values in $E$ such that $\epsilon_{i}^{\prime} \circ \operatorname{rec}_{K}(p)=0$ and $\left.\epsilon_{i}^{\prime} \circ \operatorname{rec}_{K}\right|_{\mathfrak{o}_{K}^{\times}}=\left.\epsilon_{i}\right|_{\mathfrak{o}_{K}^{\times}}$. Then there are $\epsilon_{i, \tau}(\tau \in \operatorname{Emb}(K, E))$ such that $\epsilon_{i}^{\prime}=\sum_{\tau \in \operatorname{Emb}(K, E)} \epsilon_{i, \tau} \psi_{\tau}$.

Lemma 8.4. For $h=1, n$ we have $\left[K_{0}: \mathbf{Q}_{p}\right] \gamma_{h, 0}=-v_{p}\left(\pi_{K}\right) \epsilon_{h}(p)$ and $\gamma_{h, \tau}=\epsilon_{h, \tau}$.

Proof. We keep to use notations in the proof of Theorem 7.2, By (7.3) and Lemma 8.3 we have

$$
\begin{aligned}
(\sigma-1)\left(\lambda_{h}\right) & =-\left(X^{-1} U_{\sigma} X\right)_{h h}+\sum_{\tau \in \operatorname{Emb}(K, E)} \epsilon_{h, \tau} \psi_{\tau}(\sigma) \\
& =-(\sigma-1) \xi_{h}-\gamma_{h, 0} \psi_{0}(\sigma)+\sum_{\tau \in \operatorname{Emb}(K, E)}\left(\epsilon_{h, \tau}-\gamma_{h, \tau}\right) \psi_{\tau}(\sigma) .
\end{aligned}
$$

Note that there exists $\omega \in \mathrm{W}\left(\overline{\mathbf{F}}_{p}\right)$ such that $\varphi(\omega)-\omega=1$, where $\mathrm{W}\left(\overline{\mathbf{F}}_{p}\right)$ is the ring of Witt vectors with coefficients in the algebraic closure of $\mathbf{F}_{p}$. Then $(\sigma-1) \omega=\psi_{0}(\sigma)$. Hence

$$
\sum_{\tau \in \operatorname{Emb}(K, E)}\left(\epsilon_{h, \tau}-\gamma_{h, \tau}\right) \psi_{\tau}(\sigma)=(\sigma-1)\left(\lambda_{h}+\xi_{h}+\gamma_{h, 0} \omega\right) .
$$

In other words, the cocycle $\sum_{\tau \in \operatorname{Emb}(K, E)}\left(\epsilon_{h, \tau}-\gamma_{h, \tau}\right) \psi_{\tau}(\sigma)$ is de Rham. By Lemma 5.2 we have $\gamma_{h, \tau}=\epsilon_{h, \tau}$ and $\lambda_{h}+\xi_{h}+\gamma_{h, 0} \omega \in E$. Then

$$
\left(\varphi^{\left[K_{0}: \mathbf{Q}_{p}\right]}-1\right) \lambda_{h}=-(\varphi-1) \xi_{h}-\gamma_{h, 0}\left(\varphi^{\left[K_{0}: \mathbf{Q}_{p}\right]}-1\right) \omega=-\left[K_{0}: \mathbf{Q}_{p}\right] \gamma_{h, 0} .
$$


By our choice of the basis $\left\{e_{1, z}, \ldots, e_{n, z}\right\}, Y_{1}=\oplus_{i=2}^{n} Z e_{i, z}$ is stable by $\varphi$. Put $Y_{n}=0$. Let $x$ be as in the proof of Theorem [7.2. By Lemma 7.1 we have $\varphi^{\left[K_{0}: \mathbf{Q}_{p}\right]} e_{h, z}=\alpha_{h, z} e_{h, z}$. Thus for $h=1, n$ we have

$$
\varphi^{\left[K_{0}: \mathbf{Q}_{p}\right]}(x)=\left(1+Z \varphi^{\left[K_{0}: \mathbf{Q}_{p}\right]}\left(\lambda_{h}\right)\right) \alpha_{h, z} e_{h} \quad\left(\bmod Y_{h}\right) .
$$

On the other hand,

$$
\begin{aligned}
\varphi^{\left[K_{0}: \mathbf{Q}_{p}\right]}(x) & =\left(1+Z v_{p}\left(\pi_{K}\right) \epsilon_{h}(p)\right) \alpha_{h, z} x \\
& =\left(1+Z v_{p}\left(\pi_{K}\right) \epsilon_{h}(p)\right) \alpha_{h, z}\left(1+Z \lambda_{h}\right) e_{h} \quad\left(\bmod Y_{h}\right) .
\end{aligned}
$$

Hence we obtain

$$
\left(\varphi^{\left[K_{0}: \mathbf{Q}_{p}\right]}-1\right) \lambda_{h}=v_{p}\left(\pi_{K}\right) \epsilon_{h}(p) .
$$

By (8.2) and (8.3) we have

$$
\left[K_{0}: \mathbf{Q}_{p}\right] \gamma_{h, 0}=-\left(\varphi^{\left[K_{0}: \mathbf{Q}_{p}\right]}-1\right) \lambda_{h}=-v_{p}\left(\pi_{K}\right) \epsilon_{h}(p),
$$

as wanted.

By Lemma 8.2 there exists some $y \in \mathbf{B}_{\log , E}^{\varphi=p}$ such that $N(y)=\xi_{1}-\xi_{n}$. Let $\bar{c}^{\prime}$ be the 1-cocycle of $G_{K}$ with values in $\mathbf{B}_{\log , E} \otimes_{K_{0} \otimes_{\mathbf{Q}_{p}} E} \mathscr{D}_{0}$ such that

$$
\bar{c}^{\prime}=c_{1,1}^{\prime} e_{1, z}^{*} \otimes e_{1, z}+c_{n, n}^{\prime} e_{n, z}^{*} \otimes e_{n, z}+c_{n, 1}^{\prime} e_{n, z}^{*} \otimes e_{1, z}
$$

with

$$
c_{1,1}^{\prime}=\gamma_{1,0} \psi_{0}+\sum_{\tau \in \operatorname{Emb}(K, E)} \gamma_{1, \tau} \psi_{\tau}, \quad c_{n, n}^{\prime}=\gamma_{n, 0} \psi_{0}+\sum_{\tau \in \operatorname{Emb}(K, E)} \gamma_{n, \tau} \psi_{\tau}
$$

and

$$
c_{n, 1}^{\prime}(\sigma)=c_{n, 1}(\sigma)-(\sigma-1) y, \quad \sigma \in G_{K} .
$$

It is easy to check that $\varphi\left(\bar{c}^{\prime}\right)=\bar{c}^{\prime}$ and $N\left(\bar{c}^{\prime}\right)=0$. Hence $\bar{c}^{\prime}$ is a 1-cocycle of $G_{K}$ with values in $\mathbf{X}_{\log }\left(\mathscr{D}_{0}\right)$.

Proposition 8.5. The image of $\left[\bar{c}^{\prime}\right]$ in $H^{1}\left(G_{K}, \mathbf{X}_{\log }\left(\mathscr{D}_{0}\right)\right)$ belongs to the kernel of

$$
H^{1}\left(G_{K}, \mathbf{X}_{\log }\left(\mathscr{D}_{0}\right)\right) \rightarrow H^{1}\left(G_{K}, \mathbf{X}_{\mathrm{dR}}\left(\mathscr{D}_{0}\right)\right)
$$


Proof. Consider the following commutative diagram

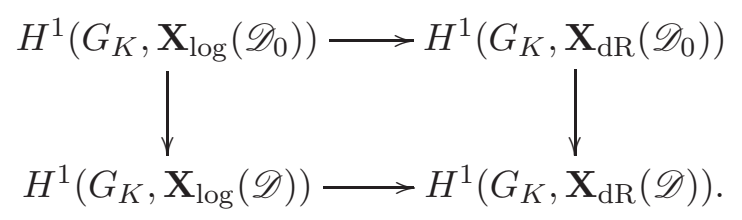

The right vertical arrow in the above diagram is injective (see [13, Corollary 2.4]). So we only need to show that the image of $\left[\bar{c}^{\prime}\right]$ in $H^{1}\left(G_{K}, \mathbf{X}_{\mathrm{dR}}(\mathscr{D})\right)$ is zero. Note that

$$
\left[\bar{c}^{\prime}\right]=[\bar{c}]-\left[c_{1, n} e_{1, z}^{*} \otimes e_{n, z}\right]=-\left[c_{1, n} e_{1, z}^{*} \otimes e_{n, z}\right]
$$

in $H^{1}\left(G_{K}, \mathbf{X}_{\mathrm{dR}}(\mathscr{D})\right)$. As the image of $\left[c_{1, n}\right]$ in $H^{1}\left(G_{K}, \mathbf{B}_{\log , E}\right)$ is zero, so is its image in $H^{1}\left(G_{K}, \mathbf{B}_{\mathrm{dR}, E} /\right.$ Fil $\left.^{f} \mathbf{B}_{\mathrm{dR}, E}\right)$, where $f$ is the smallest integer such that $e_{1, z}^{*} \otimes e_{n, z} \in \mathrm{Fil}^{-f} \mathscr{D}_{K}$. Hence, the image of $\left[\bar{c}^{\prime}\right]$ in $H^{1}\left(G_{K}, \mathbf{X}_{\mathrm{dR}}(\mathscr{D})\right)$ is zero.

Now, applying Lemma 5.3 to $\mathscr{D}_{0}$ with $f_{1}=e_{n, z}^{*} \otimes e_{1, z}, f_{2}=e_{1, z}^{*} \otimes e_{1, z}$ and $f_{3}=e_{n, z}^{*} \otimes e_{n, z}$, we get

$$
\gamma_{n, 0}-\gamma_{1,0}=\sum_{\tau \in \operatorname{Emb}(K, E)} \mathcal{L}_{\tau}\left(\gamma_{n, \tau}-\gamma_{1, \tau}\right)
$$

Hence, by Lemma 8.4 we have

$$
\frac{v_{p}\left(\pi_{K}\right)}{\left[K_{0}: \mathbf{Q}_{p}\right]}\left(\epsilon_{n}(p)-\epsilon_{1}(p)\right)+\sum_{\tau \in \operatorname{Emb}(K, E)} \mathcal{L}_{\tau}\left(\epsilon_{n, \tau}-\epsilon_{1, \tau}\right)=0 .
$$

As $\frac{\mathrm{d} \delta_{h}(p)}{\delta_{h}(p)}=\epsilon_{h}(p) \mathrm{d} Z$ and $\mathrm{d} \vec{w}\left(\epsilon_{h}\right)=\left(\epsilon_{h, \tau} \mathrm{d} Z\right)_{\tau}$, we obtain

$$
\frac{1}{\left[K: \mathbf{Q}_{p}\right]}\left(\frac{\mathrm{d} \delta_{n}(p)}{\delta_{n}(p)}-\frac{\mathrm{d} \delta_{1}(p)}{\delta_{1}(p)}\right)+\overrightarrow{\mathcal{L}}_{\mathcal{F}} \cdot\left(\mathrm{d} \vec{w}\left(\delta_{n}\right)-\mathrm{d} \vec{w}\left(\delta_{1}\right)\right)=0,
$$

as desired. This finishes the proof of Theorem 1.2 .

\section{References}

1. D. Benois, A generalization of Greenberg's $\mathscr{L}$-invariant, Amer. J. Math., 133 (2011), 1573-1632. 
2. L. Berger, Représentations p-adiques et équations différentielles, Invent. Math., 148 (2002), 219-284.

3. L. Berger, Construction de $(\varphi, \Gamma)$-modules: représentations $p$-adiques et $B$-paires, Algebra Number Theory, 2 (2008), 91-120.

4. L. Berger, Trianguline representations, Bull. Lond. Math. Soc., 43 (2011), no. 4, 619635.

5. P. Colmez, Représentations triangulines de dimension 2. Astérisque, 319 (2008), 213258.

6. P. Colmez, Invariants $\mathcal{L}$ et dérivées de valeurs propres de Frobenius. Astérisque, 331 (2010), 13-28.

7. J.-M. Fontaine, Le corps des périodes p-adiques, Astérisque, 223 (1994), 59-111.

8. R. Greenberg, G. Steven, p-adic L-functions and p-adic periods of modular forms, Invent. Math., 111 (1993), 407-447.

9. B. Mazur, The theme of $p$-adic variation, Mathematics: frontiers and perspectives, Amer. Math. Soc., Providence 2000, 433-459.

10. B. Mazur, J. Tate, J. Teitelbaum, On $p$-adic analogs of the conjectures of Birch and Swinnerton-Dyer, Invent. Math., 84 (1986), 1-48.

11. K. Nakamura, Classification of two-dimensional split trianguline representations of p-adic fields, Compos. Math., 145 (2009), 865-914.

12. J. Pottharst, The $\mathcal{L}$-invariant, the dual $\mathcal{L}$-invariant, and families, Ann. Math. Qué., 40 (2016), 159-165.

13. B. Xie, Derivatives of Frobenius and Derivatives of Hodge-Tate weights. To appear in Acta Mathematica Sinica, a special issue on Arithmetic Algebraic Geometry, edited by $\mathrm{Fu}$, Liu, Tian and $\mathrm{Xu}$.

14. Y. Zhang, $\mathcal{L}$-invariants and logarithmic derivatives of eigenvalues of Frobenius, Science China Mathematics, 57 (2014), 1587-1604. 